\title{
Moments of zeta and correlations of divisor-sums: IV
}

\author{
Brian Conrey ${ }^{1,2}$ and Jonathan P. Keating ${ }^{2^{*}}$
}

\section{*Correspondence:}

j.p.keating@bristol.ac.uk

${ }^{2}$ School of Mathematics,

University of Bristol, Bristol BS8

$1 T W$, UK

Full list of author information is

available at the end of the article

\begin{abstract}
In this series we examine the calculation of the $2 \mathrm{kth}$ moment and shifted moments of the Riemann zeta-function on the critical line using long Dirichlet polynomials and divisor correlations. The present paper begins the general study of what we call Type II sums which utilize a circle method framework and a convolution of shifted convolution sums to obtain all of the lower order terms in the asymptotic formula for the mean square along $[T, 2 T]$ of a Dirichlet polynomial of length up to $T^{3}$ with divisor functions as coefficients.
\end{abstract}

\section{Background}

This paper is part 4 of a sequence of papers devoted to understanding how to conjecture all of the integral moments of the Riemann zeta-function from a number theoretic perspective. The method is to approximate $\zeta(s)^{k}$ by a long Dirichlet polynomial and then compute the mean square of the Dirichlet polynomial (c.f. [9]). There will be many offdiagonal terms and it is the care of these that is the concern of these papers. In particular it is necessary to treat the off-diagonal terms by a method invented by Bogomolny and Keating [1,2]. Our perspective on this method is that it is most properly viewed as a multi-dimensional Hardy-Littlewood circle method.

In part 3 [7] we considered the type I off diagonal terms from a general perspective. Now we look at the simplest type II sums.

The formula we obtain is in complete agreement with all of the main terms predicted by the recipe of [3] (and in particular, with the leading order term conjectured in [10]).

\section{Shifted moments}

We are interested in developing a number theoretic approach to the moments of the Riemann zeta-function on the critical line, in particular to the general "shifted" moment given by

$$
I_{A, B}^{\psi}(T)=\int_{0}^{\infty} \psi\left(\frac{t}{T}\right) \prod_{\alpha \in A} \zeta(s+\alpha) \prod_{\beta \in B} \zeta(1-s+\beta) d t
$$

where $\psi$ is a smooth function with compact support, say $\psi \in C^{\infty}[1,2]$ and $s=1 / 2+i t$ and $A$ and $B$ are sets of small complex numbers, referred to as the shifts. It is useful to consider as well the general shifted moment of a long Dirichlet polynomial. To express this we first introduce the generalized divisor function $\tau_{A}(n)$ by way of its generating function:

(c) 2016 The Author(s). This article is distributed under the terms of the Creative Commons Attribution 4.0 International License (http://creativecommons.org/licenses/by/4.0/), which permits unrestricted use, distribution, and reproduction in any medium, provided you give appropriate credit to the original author(s) and the source, provide a link to the Creative Commons license, and indicate if changes were made. 


$$
\prod_{\alpha \in A} \zeta(s+\alpha)=\sum_{n=1}^{\infty} \frac{\tau_{A}(n)}{n^{s}}=: D_{A}(s) .
$$

Then we let

$$
\mathcal{D}_{A}(s ; X)=\sum_{n \leq X} \frac{\tau_{A}(n)}{n^{s}}
$$

and consider

$$
\begin{aligned}
I_{A, B}^{\psi}(T ; X): & =\int_{0}^{\infty} \psi\left(\frac{t}{T}\right) D_{A}(s ; X) D_{B}(1-s ; X) d t \\
& =T \sum_{m, n \leq X} \frac{\tau_{A}(m) \tau_{B}(n) \hat{\psi}\left(\frac{T}{2 \pi} \log \frac{m}{n}\right)}{\sqrt{m n}} .
\end{aligned}
$$

The recipe [3] tells us how to predict the behaviour of these moments. Firstly, we conjecture that

$$
\begin{aligned}
I_{A, B}^{\psi}(T)= & T \int_{0}^{\infty} \psi(t) \sum_{\substack{U \subset A, V \subset B \\
|U|=|V|}}\left(\frac{t T}{2 \pi}\right)^{-\sum_{\substack{\alpha \in U \\
\beta \in V}}(\alpha+\beta)} \\
& \times \mathcal{B}\left(A-U+V^{-}, B-V+U^{-}\right) d t+o(T)
\end{aligned}
$$

where $\mathcal{B}$ is given by

$$
\mathcal{B}(A, B)=\sum_{n=1}^{\infty} \frac{\tau_{A}(n) \tau_{B}(n)}{n}
$$

in the case that this series converges (for example if $\Re \alpha, \Re \beta>0$ for all $\alpha \in A$ and $\beta \in B$ ) and is given by analytic continuation otherwise. An alternate expression is $\mathcal{B}(A, B)=$ $\mathcal{A}(A, B) \mathcal{Z}(A, B)$ where

$$
Z(A, B):=\prod_{\substack{\alpha \in A \\ \beta \in B}} \zeta(1+\alpha+\beta)
$$

and $\mathcal{A}(A, B)$ is a product over primes that converges nicely in the domains under consideration (see below). We have used an unconventional notation here; by $A-U+V^{-}$we mean the following: start with the set $A$ and remove the elements of $U$ and then include the negatives of the elements of $V$. We think of the process as "swapping" equal numbers of elements between $A$ and $B$; when elements are removed from $A$ and put into $B$ they first get multiplied by -1 . We keep track of these swaps with our equal-sized subsets $U$ and $V$ of $A$ and $B$; and when we refer to the "number of swaps" in a term we mean the cardinality $|U|$ of $U$ (or, since they are of equal size, of $V$ ).

The Euler product $\mathcal{A}$ is given by

$$
\mathcal{A}(A, B)=\prod_{p} Z_{p}(A, B) \int_{0}^{1} \mathcal{A}_{p, \theta}(A, B) d \theta,
$$

where $z_{p}(x):=\left(1-p^{-x}\right)^{-1}, Z_{p}(A, B)=\prod_{\substack{\alpha \in A \\ \beta \in B}} z_{p}(1+\alpha+\beta)^{-1}$ and

$$
\mathcal{A}_{p, \theta}(A, B):=\prod_{\alpha \in A} z_{p,-\theta}\left(\frac{1}{2}+\alpha\right) \prod_{\beta \in B} z_{p, \theta}\left(\frac{1}{2}+\beta\right)
$$

with $z_{p, \theta}(x):=\left(1-e(\theta) p^{-x}\right)^{-1}$. 
The technique we are developing in the present series of papers is to approach our moment problem (1) through the moments $I_{A, B}^{\psi}(T ; X)$ of long Dirichlet polynomials for various ranges of $X$. The recipe of [3] also leads to a conjectural formula for $I_{A, B}^{\psi}(T ; X)$. To explain this we begin with Perron's formula

$$
D_{A}(s ; X)=\frac{1}{2 \pi i} \int_{w} \frac{X^{w}}{w} D_{A_{w}}(s) d w
$$

where we use the convenient notation

$$
A_{w}=\{\alpha+w: \alpha \in A\} .
$$

Thus, we have

$$
I_{A, B}^{\psi}(T ; X)=\frac{1}{(2 \pi i)^{2}} \iint_{z, w} \frac{X^{z+w}}{z w} I_{A_{w}, B_{z}}^{\psi}(T) d w d z .
$$

We insert the conjecture above from the recipe and expect that

$$
\begin{aligned}
I_{A, B}^{\psi}(T ; X)= & T \int_{0}^{\infty} \psi(t) \frac{1}{(2 \pi i)^{2}} \iint_{z, w} \frac{X^{z+w}}{z w} \sum_{\substack{U \subset A, V \subset B \\
|U|=|V|}}\left(\frac{t T}{2 \pi}\right)^{-\sum_{\substack{\alpha \in U \\
\beta \in V}}(\alpha+w+\beta+z)} \\
& \times \mathcal{B}\left(A_{w}-U_{w}+V_{z}^{-}, B_{z}-V_{z}+U_{w}^{-}\right) d w d z d t+o(T) .
\end{aligned}
$$

We have done a little simplification in this expression: instead of writing $U \subset A_{w}$ we have written $U \subset A$ and changed the exponent of $(t T / 2 \pi)$ accordingly.

Notice that there is a factor $\left(X / T^{|U|}\right)^{w+z}$ here. As mentioned above we refer to $|U|$ as the number of "swaps" in the recipe, and now we see more clearly the role it plays; in the terms above for which $X<T^{|U|}$ we move the path of integration in $w$ or $z$ to $+\infty$ so that the factor $\left(X / T^{|U|}\right)^{w+z} \rightarrow 0$ and the contribution of such a term is 0 . Thus, the size of $X$ determines how many "swaps" we must keep track of.

Our principal aim in this series of papers is to evaluate $I_{A, B}^{\psi}(T ; X)$ directly using a conjecture for the correlations of $\tau_{A}(n)$ and then to compare with the above formula coming from the recipe of [3]. In [5] and [7] we considered the situation of 0 swaps which leads to the usual "diagonal" terms and 1 swap which corresponds to the usual "shifted divisor" problem. In [6] we considered a special case of 2 swaps. Now we look at the general case of two swaps. This means that we are interested in the terms for which $X>T^{2}$ and for which $|U|=|V|=2$.

It is helpful to review the result of [7] before proceeding. The mathematical content of that paper is basically a conjecture and a theorem. First of all let $\epsilon>0$ be a small fixed number for this discussion and let $|\alpha|,|\beta|<\epsilon$ for all $\alpha \in A$ and $\beta \in B$. The conjecture is about the analytic continuation of

$$
\mathcal{S}_{A, B}(s, h):=\sum_{m=1}^{\infty} \frac{\tau_{A}(m) \tau_{B}(m+h)}{m^{s}}
$$

and the sum of the residues near 1 of this:

$$
\mathcal{R}_{A, B}(y ; h):=\sum_{|s-1|<\epsilon} \operatorname{Res} S_{A, B}(s, h) y^{s-1}
$$

where we intend this notation to mean that $\mathcal{R}_{A, B}(y ; h)$ is the sum of the residues of $S_{A, B}(s, h) y^{s-1}$ over all of the poles in $|s-1|<\epsilon$. Let

$$
D_{A}\left(s, e\left(\frac{1}{q}\right)\right):=\sum_{m=1}^{\infty} \frac{\tau_{A}(m) e\left(\frac{m}{q}\right)}{m^{s}}
$$


and

$$
\mathcal{R}_{A}(y, q)=\sum_{|s-1|<\epsilon} \operatorname{Res} D_{A}\left(s, e\left(\frac{1}{q}\right)\right) y^{s-1}
$$

be the sum of the residues near $s=1$, i.e. including poles at $s=1-\alpha$ for $\alpha \in A$. Let

$$
\mathcal{R}_{A, B}^{*}(y ; h):=\sum_{q=1}^{\infty} r_{q}(h) \mathcal{R}_{A}(y, q) \mathcal{R}_{B}(y, q)
$$

where $r_{q}(h)$ is the Ramanujan sum.

Conjecture 1 We conjecture for each fixedh $>0$ that $S_{A, B}(s, h)$ has a meromorphic continuation toßs $>\frac{1}{2}+\epsilon$ with all poles only in the region $|s-1|<\epsilon$ and that

$$
\mathcal{R}_{A, B}(y ; h)=\mathcal{R}_{A, B}^{*}(y ; h) .
$$

The above is essentially the obvious pole structure that one would conjecture by using the $\delta$-method for example.

Now we briefly describe the calculation of [7]. We evaluate

$$
\sum_{\substack{m, n \leq X \\ m \neq n}} \frac{\tau_{A}(m) \tau_{B}(n)}{\sqrt{m n}} \hat{\psi}\left(\frac{T}{2 \pi} \log \frac{m}{n}\right)
$$

as

$$
2 \sum_{h>0} \int_{T}^{X}\left\langle\tau_{A}(m) \tau_{B}(m+h)\right\rangle_{m \sim u} \hat{\psi}\left(\frac{T h}{2 \pi u}\right) \frac{d u}{u}
$$

which we evaluate by differentiating Perron's formula with respect to $u$ and then moving the $s$-contour to the left to give

$$
2 \sum_{h>0} \int_{T}^{X} \mathcal{R}_{A, B}(u, h) \hat{\psi}\left(\frac{T h}{2 \pi u}\right) \frac{d u}{u}
$$

We make the change of variable $v=\frac{T h}{2 \pi u}$ and rewrite this as

$$
2 \int_{0}^{\infty} \hat{\psi}(v) \sum_{h \leq \frac{2 \pi X_{v}}{T}} \mathcal{R}_{A, B}\left(\frac{T h}{2 \pi v}, h\right) \frac{d v}{v}
$$

At this point we replace $\mathcal{R}$ by $\mathcal{R}^{*}$ and have

$$
2 \int_{0}^{\infty} \hat{\psi}(v) \sum_{h \leq \frac{2 \pi X v}{T}} \sum_{q=1}^{\infty} r_{q}(h) \mathcal{R}_{A}\left(\frac{T h}{2 \pi v}, q\right) \mathcal{R}_{B}\left(\frac{T h}{2 \pi v}, q\right) \frac{d v}{v} .
$$

Now

$$
r_{q}(h)=\sum_{\substack{d|h \\ d| q}} d \mu(q / d)
$$

so, replacing $h$ by $h d$ and $q$ by $q d$ the above is

$$
2 \int_{0}^{\infty} \hat{\psi}(v) \sum_{q=1}^{\infty} \mu(q) \sum_{h d \leq \frac{2 \pi X_{v}}{T}} d \mathcal{R}_{A}\left(\frac{T h d}{2 \pi v}, q d\right) \mathcal{R}_{B}\left(\frac{T h d}{2 \pi v}, q d\right) \frac{d v}{v} .
$$

Now we express this using Cauchy's theorem as

$$
\begin{aligned}
& 2 \int_{0}^{\infty} \hat{\psi}(v) \sum_{q=1}^{\infty} \frac{\mu(q)}{(2 \pi i)^{3}} \iiint_{\substack{|\uparrow s=2\\
| z-1|<\epsilon\\
| z-1 \mid<\epsilon}} X^{s} \sum_{h, d=1}^{\infty}\left(\frac{T h d}{2 \pi v}\right)^{z+w-s-2} d \\
& \quad \times \mathcal{D}_{A}\left(w, e\left(-\frac{1}{q d}\right)\right) \mathcal{D}_{B}\left(z, e\left(-\frac{1}{q d}\right)\right) \frac{d v}{v} d z d w \frac{d s}{s} .
\end{aligned}
$$


Now we replace the sum over $h$ by $\zeta(2+s-w-z)$ and the integral over $v$ by

$$
\frac{\chi(w+z-s-1)}{2} \int_{0}^{\infty} \psi(t) t^{z+w-s-2} d t .
$$

This leads to

$$
\begin{aligned}
& \int_{0}^{\infty} \psi(t) \sum_{q=1}^{\infty} \frac{\mu(q)}{(2 \pi i)^{3}} \iiint_{\substack{|\Re s=2\\
| w-1|<\epsilon\\
| z-1 \mid<\epsilon}} X^{s} \zeta(w+z-s-1) \sum_{d=1}^{\infty} d\left(\frac{T d t}{2 \pi}\right)^{z+w-s-2} \\
& \quad \times \mathcal{D}_{A}\left(w, e\left(-\frac{1}{q d}\right)\right) \mathcal{D}_{B}\left(z, e\left(-\frac{1}{q d}\right)\right) d t d z d w d s .
\end{aligned}
$$

Upon comparison with the recipe we have the identity

\section{Theorem 1}

$$
\begin{aligned}
& \operatorname{Res}_{\substack{w=1-\alpha \\
z=1-\beta}} \sum_{q=1}^{\infty} \mu(q) \sum_{d=1}^{\infty} d^{z+w-1} \zeta(w+z-1) \mathcal{D}_{A}\left(w, e\left(-\frac{1}{q d}\right)\right) \mathcal{D}_{B}\left(z, e\left(-\frac{1}{q d}\right)\right) \\
& \quad=\mathcal{B}\left(A^{\prime} \cup\{-\beta\}, B^{\prime} \cup\{-\alpha\}\right) .
\end{aligned}
$$

Theorem 1 follows from the identity stated at the end of Sect. 3 of [7] and the fact that the singular part of $\mathcal{D}_{A}\left(s, e\left(\frac{1}{q}\right)\right)$ is the same as $q^{-s} \prod_{\alpha \in A} \zeta(s+\alpha) G_{A}(s, q)$, as proved in [4].

We call this theorem the "analytic version of the general shifted divisor sum." In this paper we prove an identity that is an analogue of Theorem 1 but for a convolution of two shifted divisor sums. This is a step forward in this process of understanding moments. The key theorem is a convolution identity

\section{Theorem 2}

$$
\begin{aligned}
& \operatorname{Res}_{\substack{w_{1}=1-\alpha_{1} \\
z_{1}=1-\beta_{1} \\
w_{2}=1-\alpha_{2} \\
z_{2}=1-\beta_{2}}} \zeta\left(w_{1}+z_{1}-1\right) \zeta\left(w_{2}+z_{2}-1\right) \sum_{\substack{(M, N)=1 \\
d_{1}, d_{2} \\
q_{1}, q_{2}}} \frac{\mu\left(q_{1}\right) \mu\left(q_{2}\right) d_{1}^{z_{1}+w_{1}-1} d_{2}^{z_{2}+w_{2}-1}}{M^{z_{1}+w_{2}-1} N^{w_{1}+z_{2}-1}} \\
& \times \mathcal{D}_{A_{1}}\left(w_{1}, e\left(-\frac{N}{q_{1} d_{1}}\right)\right) \mathcal{D}_{A_{2}}\left(w_{2}, e\left(-\frac{M}{q_{2} d_{2}}\right)\right) \\
& \times \mathcal{D}_{B_{1}}\left(z_{1}, e\left(-\frac{M}{q_{1} d_{1}}\right)\right) \mathcal{D}_{B_{2}}\left(z_{2}, e\left(-\frac{N}{q_{2} d_{2}}\right)\right) \\
& =\mathcal{B}\left(A^{\prime \prime} \cup\left\{-\beta_{1},-\beta_{2}\right\}, B^{\prime \prime} \cup\left\{-\alpha_{1},-\alpha_{2}\right\}\right) \text {. }
\end{aligned}
$$

Theorem 2 follows from Sect. 11 because if $(a, q)=1$ then the singular part of $\mathcal{D}_{A}\left(s, e\left(\frac{a}{q}\right)\right)$ is identical to that of $q^{-s} \prod_{\alpha \in A} \zeta(s+\alpha) G_{A}(s, q)$.

A particularly interesting feature of this theorem is the appearance of the sum over $M$ and $N$. It is these parameters which prompt us to liken this calculation to a circle method calculation. Basically the $M$ and $N$ make their appearance because of a splitting of the equation $m_{1} m_{2}-n_{1} n_{2}=h$ into a pair of equations where $m_{1} / n_{1} \approx M / N \approx n_{2} / m_{2}$ which gives $M m_{1}-N n_{1}=h_{1}, N m_{2}-M n_{2}=h_{2}$. This is the fundamental new idea of the paper.

In the next section of this paper we present the basic set up, which involves a convolution of two shifted divisor sums. In Sect. 4, 5 and 6 we deal with the semi-diagonal case where one of the shifted divisor sums is degenerate. In Sect. 7, 8 and 9 we motivate heuristically the identity of Theorem 2 . This identity is sufficiently complicated that we find it convenient to recast it as an equality of certain power series. Sect. 10 and 11 are devoted to the rigorous proof of this identity. 


\section{Type II convolution sums}

To proceed we approach the moment $I_{A, B}^{\psi}(T ; X)$ through arithmetic means. To do this, we consider a convolution of shifted correlation sums.

We first make use of the fact that if $A=A_{1} \cup A_{2}$ and $B=B_{1} \cup B_{2}$ then $\tau_{A}$ and $\tau_{B}$ are convolutions: $\tau_{A}=\tau_{A_{1}} * \tau_{A_{2}}$ and $\tau_{B}=\tau_{B_{1}} * \tau_{B_{2}}$. We are thus interested in

$$
\begin{aligned}
\mathcal{O}_{I I}= & \sum_{\substack{m_{1} m_{2}, n_{1} n_{2} \leq X \\
0<\left|m_{1} m_{2}-n_{1} n_{2}\right|<m_{1} m_{2} / \tau}} \frac{\tau_{A_{1}}\left(m_{1}\right) \tau_{A_{2}}\left(m_{2}\right) \tau_{B_{1}}\left(n_{1}\right) \tau_{B_{2}}\left(n_{2}\right)}{m_{1} m_{2}} \\
& \times \hat{\psi}\left(\frac{T}{2 \pi} \log \left(\left(n_{1} n_{2}\right) /\left(m_{1} m_{2}\right)\right)\right) .
\end{aligned}
$$

Now we embark on a discrete analog of the circle method which basically consists of approximating a ratio, say $m_{1} / n_{1}$ by a rational number with a small denominator, say $M / N$, and then sum all of the terms with $m_{1} / n_{1}$ close to $M / N$.

To this end we introduce a parameter $Q$ and subdivide the interval $[0,1]$ into Farey intervals associated with the fractions $M / N$ with $1 \leq M \leq N \leq Q$ and $(M, N)=1$ from the Farey sequence $\mathcal{F}_{Q}$; see [6] for details. We define

$$
h_{1}:=m_{1} N-n_{1} M
$$

and

$$
h_{2}:=m_{2} M-n_{2} N
$$

We have

$$
m_{1} m_{2} M N-n_{1} n_{2} M N=h_{1} m_{2} M+h_{2} m_{1} N-h_{1} h_{2}
$$

so that

and

$$
\frac{m_{1} m_{2}-n_{1} n_{2}}{m_{1} m_{2}}=\frac{h_{1}}{m_{1} N}+\frac{h_{2}}{m_{2} M}-\frac{h_{1} h_{2}}{m_{1} m_{2} M N}
$$

$$
\log \frac{n_{1} n_{2}}{m_{1} m_{2}}=\frac{h_{1}}{m_{1} N}+\frac{h_{2}}{m_{2} M}+O\left(\frac{h_{1} h_{2}}{m_{1} m_{2} M N}\right) .
$$

The error term is negligible so we have now arranged the sum as

$$
\sum_{\substack{M \leq N \leq Q \\(M, N)=1}} \sum_{h_{1}, h_{2}} \sum_{\substack{m_{1} m_{2} \leq X \\\left(*_{1}\right),\left(*_{2}\right)}} \frac{\tau_{A_{1}}\left(m_{1}\right) \tau_{A_{2}}\left(m_{2}\right) \tau_{B_{1}}\left(n_{1}\right) \tau_{B_{2}}\left(n_{2}\right)}{m_{1} m_{2}} \hat{\psi}\left(\frac{T h_{1}}{2 \pi m_{1} N}+\frac{T h_{2}}{2 \pi m_{2} M}\right)
$$

where

$$
\left(*_{1}\right): m_{1} N-n_{1} M=h_{1} \quad \text { and } \quad\left(*_{2}\right): m_{2} M-n_{2} N=h_{2}
$$

Note that for a given $m_{1}, n_{1}$ and $h_{1}$ the condition $\left(*_{1}\right)$ implies that $m_{1} / n_{1} \in \mathcal{M}_{M, N}$ so we don't need to write that condition.

\section{The case of $h_{2}=0$}

We remark first of all that the terms with $h_{1}=h_{2}=0$ are precisely the diagonal terms. Now we consider what happens if $h_{2}=0$ and $h_{1} \neq 0$. We call this a "semi-diagonal" term after [1].

If $h_{2}=0$ then $m_{2} M=n_{2} N$. Since $(M, N)=1$ it follows that $m_{2}=N \ell$ and $n_{2}=M \ell$ for some $\ell$. Thus we have

$$
\sum_{\substack{M \leq N \\(M, N)=1}} \phi\left(\frac{M}{Q}\right) \phi\left(\frac{N}{Q}\right) \sum_{h_{1}} \sum_{\substack{\left.m_{1}, n_{1}, \ell \\()_{1}\right) \\ n_{1} \geq\left|h_{1}\right| Q}} \frac{\tau_{A_{1}}\left(m_{1}\right) \tau_{A_{2}}(N \ell) \tau_{B_{1}}\left(n_{1}\right) \tau_{B_{2}}(M \ell)}{m_{1} N \ell} \hat{\psi}\left(\frac{T h_{1}}{2 \pi m_{1} N}\right)
$$


where

$$
\left(*_{1}\right): m_{1} N-n_{1} M=h_{1} .
$$

In general, with $*: m N-n M=h$, we expect by the delta-method that

$$
\begin{aligned}
& \left\langle\tau_{A}(m) \tau_{B}(n)\right\rangle_{m=u}^{(*)} \sim \sum_{\substack{\alpha \in A \\
\beta \in B}} u^{-\alpha-\beta} M^{-1+\beta} N^{-\beta} Z\left(A_{-\alpha}^{\prime}\right) Z\left(B_{-\beta}^{\prime}\right) \\
& \times \sum_{d \mid h} \frac{1}{d^{1-\alpha-\beta}} \sum_{q} \frac{\mu(q)(q d, M)^{1-\beta}(q d, N)^{1-\alpha}}{q^{2-\alpha-\beta}} \\
& \times G_{A}\left(1-\alpha, \frac{q d}{(q d, N)}\right) G_{B}\left(1-\beta, \frac{q d}{(q d, M)}\right),
\end{aligned}
$$

where $G$ is a multiplicative function for which

$$
G_{A}\left(1-\alpha, p^{r}\right)=\prod_{\hat{\alpha} \in A^{\prime}}\left(1-\frac{1}{p^{1+\hat{\alpha}-\alpha}}\right) \sum_{j=0}^{\infty} \frac{\tau_{A^{\prime}}\left(p^{j+r}\right)}{p^{j(1-\alpha)}}
$$

with $A^{\prime}=A-\{\alpha\}$ and where

$$
Z(A)=\prod_{a \in A} \zeta(1+a)
$$

\section{A diversion}

The ensuing calculations are about to become (more) complicated largely due to arithmetic factors. We pause in the calculation to show what the calculations look like without the arithmetic factors. That should help the reader when we complete this calculation in the next section. Basically we ignore the terms with $q \geq 2$ and we replace $G_{A}(1-\alpha, d)$ by $\tau_{A^{\prime}}(d)$.

Altogether we now have

$$
\begin{aligned}
& \sum_{\substack{\alpha \in A_{1} \\
\beta \in B_{1}}} Z\left(\left(A_{1}^{\prime}\right)_{-\alpha}\right) Z\left(\left(B_{1}^{\prime}\right)_{-\beta}\right) \sum_{\substack{M \leq N \\
(M, N)=1}} \phi\left(\frac{M}{Q}\right) \phi\left(\frac{N}{Q}\right) \sum_{\ell, h_{1}} \frac{\tau_{A_{2}}(\ell) \tau_{B_{2}}(\ell)}{\ell} \\
& \quad \times \int_{u \leq \frac{X}{N \ell}} \sum_{d \mid h_{1}} \frac{(d, N)^{1-\alpha}(d, M)^{1-\beta} \tau_{A_{1}^{\prime}}\left(\frac{d}{(d, N)}\right) \tau_{B_{1}^{\prime}}\left(\frac{d}{(d, M)}\right) \tau_{A_{2}}(N) \tau_{B_{2}}(M)}{M^{1-\beta} N^{1+\beta} u^{\alpha+\beta} d^{1-\alpha-\beta}} \hat{\psi}\left(\frac{T h_{1}}{2 \pi u N}\right) \frac{d u}{u} .
\end{aligned}
$$

We make the substitution

$$
v=\frac{T h_{1}}{2 \pi u N} .
$$

The above is

$$
\begin{aligned}
& \sum_{\substack{\alpha \in A_{1} \\
\beta \in B_{1}}} Z\left(\left(A_{1}^{\prime}\right)_{-\alpha}\right) Z\left(\left(B_{1}^{\prime}\right)_{-\beta}\right) \sum_{\substack{M \leq N \\
(M, N)=1}} \phi\left(\frac{M}{Q}\right) \phi\left(\frac{N}{Q}\right) \sum_{\ell, h_{1}} \frac{\tau_{A_{2}}(\ell) \tau_{B_{2}}(\ell)}{\ell} \\
& \times \int_{v \geq \frac{T h_{1} \ell}{2 \pi X}} \sum_{d \mid h_{1}} \frac{(d, N)^{1-\alpha}(d, M)^{1-\beta} \tau_{A_{1}^{\prime}}\left(\frac{d}{(d, N)}\right) \tau_{B_{1}^{\prime}}\left(\frac{d}{(d, M)}\right) \tau_{A_{2}}(N) \tau_{B_{2}}(M)}{M^{1-\beta} N^{1+\beta}\left(\frac{T h_{1}}{2 \pi v N}\right)^{\alpha+\beta}} d^{1-\alpha-\beta}(v) \frac{d v}{v} .
\end{aligned}
$$


Now we switch the sums around; replacing $h_{1}$ by $h_{1} d$ and bringing the sum over $h_{1}$ and $\ell$ to the inside, we have

$$
\begin{aligned}
& \sum_{\substack{\alpha \in A_{1} \\
\beta \in B_{1}}}\left(\frac{T}{2 \pi}\right)^{-\alpha-\beta} Z\left(\left(A_{1}^{\prime}\right)_{-\alpha}\right) Z\left(\left(B_{1}^{\prime}\right)_{-\beta}\right) \sum_{\substack{M \leq N \\
(M, N)=1}} \frac{\phi\left(\frac{M}{Q}\right) \phi\left(\frac{N}{Q}\right) \tau_{A_{2}}(N) \tau_{B_{2}}(M)}{M^{1-\beta} N^{1-\alpha}} \\
& \quad \times \int_{v} \frac{\hat{\psi}(v)}{v^{1-\alpha-\beta}} \sum_{d \ell h_{1} \leq \frac{2 \pi X_{v}}{T}} \frac{(d, N)^{1-\alpha}(d, M)^{1-\beta} \tau_{A_{1}^{\prime}}\left(\frac{d}{(d, N)}\right) \tau_{B_{1}^{\prime}}\left(\frac{d}{(d, M)}\right) \tau_{A_{2}}(\ell) \tau_{B_{2}}(\ell)}{d \ell h_{1}^{\alpha+\beta}} d v .
\end{aligned}
$$

Using Perron's formula we write this as

$$
\begin{aligned}
& \sum_{\substack{\alpha \in A_{1} \\
\beta \in B_{1}}}\left(\frac{T}{2 \pi}\right)^{-\alpha-\beta} Z\left(\left(A_{1}^{\prime}\right)_{-\alpha}\right) Z\left(\left(B_{1}^{\prime}\right)_{-\beta}\right) \sum_{\substack{M \leq N \\
(M, N)=1}} \frac{\phi\left(\frac{M}{Q}\right) \phi\left(\frac{N}{Q}\right) \tau_{A_{2}}(N) \tau_{B_{2}}(M)}{M^{1-\beta} N^{1-\alpha}} \\
& \quad \times \int_{v} \frac{\hat{\psi}(v)}{v^{1-\alpha-\beta}} \frac{1}{2 \pi i} \int_{(2)} \sum_{d, \ell, h_{1}} \frac{(d, N)^{1-\alpha}(d, M)^{1-\beta} \tau_{A_{1}^{\prime}}\left(\frac{d}{(d, N)}\right) \tau_{B_{1}^{\prime}}\left(\frac{d}{(d, M)}\right) \tau_{A_{2}}(\ell) \tau_{B_{2}}(\ell)}{d^{s+1} \ell^{s+1} h_{1}^{s+\alpha+\beta}} \\
& \quad \times\left(\frac{2 \pi X v}{T}\right)^{s} \frac{d s}{s} d v .
\end{aligned}
$$

The sum over $\ell$ and $h_{1}$ here is essentially

$$
\zeta(s+\alpha+\beta) Z\left(\left(A_{2}\right)_{s}, B_{2}\right) .
$$

The sum over $d, M$ and $N$ we evaluate to a first approximation by looking at the polar parts of

$$
\sum_{\substack{d, M, N \\(M, N)=1}} \frac{(d, M)^{1-\beta}(d, N)^{1-\alpha} \tau_{A_{1}^{\prime}}\left(\frac{d}{(d, N)}\right) \tau_{B_{1}^{\prime}}\left(\frac{d}{(d, M)}\right) \tau_{A_{2}}(N) \tau_{B_{2}}(M)}{d^{1+s} M^{1-\beta} N^{1-\alpha}} ;
$$

these are calculated with the help of the following table:

\begin{tabular}{|l|l|l|l|l|}
\hline$d$ & $M$ & $N$ & Euler term & $Z$ - factor \\
\hline$p$ & 1 & 1 & $\tau_{A_{1}^{\prime}}(p) \tau_{B_{1}^{\prime}}(p) / p^{1+s}$ & $Z\left(\left(A_{1}^{\prime}\right)_{s}, B_{1}^{\prime}\right)$ \\
\hline 1 & $p$ & 1 & $\tau_{B_{2}}(p) / p^{1-\beta}$ & $Z\left(B_{2},\{-\beta\}\right)$ \\
\hline 1 & 1 & $p$ & $\tau_{A_{2}}(p) / p^{1-\alpha}$ & $Z\left(A_{2},\{-\alpha\}\right)$ \\
\hline$p$ & 1 & $p$ & $\tau_{B_{1}^{\prime}}(p) \tau_{A_{2}}(p) / p^{1+s}$ & $Z\left(B_{1}^{\prime},\left(A_{2}\right)_{s}\right)$ \\
\hline$p$ & $p$ & 1 & $\tau_{A_{1}^{\prime}}(p) \tau_{B_{2}}(p) / p^{1+s}$ & $Z\left(\left(A_{1}^{\prime}\right)_{s}, B_{2}\right)$ \\
\hline
\end{tabular}

We take the product of all of these $Z$ factors. Now the $v$-integral is

$$
\int_{v} \frac{\hat{\psi}(v)}{v^{1-s-\alpha-\beta}} d v=(1 / 2) \chi(1-s-\alpha-\beta) \int_{0}^{\infty} \psi(t) t^{-s-\alpha-\beta} d t .
$$

Note that

$$
\chi(1-s-\alpha-\beta) \zeta(s+\alpha+\beta)=\zeta(1-s-\alpha-\beta) .
$$

If we include the factors $Z\left(\left(A_{2}\right)_{s}, B_{2}\right), Z\left(\left(A_{1}^{\prime}\right)_{-\alpha}\right) Z\left(\left(B_{1}^{\prime}\right)_{-\beta}\right)$, and $Z(\{-s-\alpha\},\{-\beta\})$ then the product of all of these $Z$-factors is

$$
Z\left(\left(A_{1}^{\prime} \cup A_{2}\right)_{s} \cup\{-\beta\}, B_{1}^{\prime} \cup B_{2} \cup\{-s-\alpha\}\right)=Z\left(\left(A^{\prime}\right)_{s} \cup\{-\beta\}, B^{\prime} \cup\{-s-\alpha\}\right) .
$$


Thus, altogether we have

$$
\sum_{\substack{\alpha \in A_{1} \\ \beta \in B_{1}}}\left(\frac{T}{2 \pi}\right)^{-\alpha-\beta} \int_{t} \frac{\psi(t)}{t^{\alpha+\beta}} \frac{1}{2 \pi i} \int_{(2)} Z\left(\left(A^{\prime}\right)_{s} \cup\{-\beta\}, B^{\prime} \cup\{-s-\alpha\}\right)\left(\frac{2 \pi X}{t T}\right)^{s} \frac{d s}{s} d t .
$$

Compare this with Eq. (4) of [7] which gives the "one-swap" terms from the recipe:

$$
\begin{aligned}
& \int_{0}^{\infty} \psi(t) \sum_{\substack{\alpha \in A \\
\beta \in B}}\left(\frac{T t}{2 \pi}\right)^{-\alpha-\beta} Z\left(\left(A^{\prime}\right)_{-\alpha}\right) Z\left(\left(B^{\prime}\right)_{-\beta}\right) \\
& \quad \times \frac{1}{2 \pi i} \int_{\Re s=4} \frac{\left(\frac{2 \pi X}{T t}\right)^{s}}{s} \mathcal{A}\left(A^{\prime} \cup\{-\beta-s\}, B_{s}^{\prime} \cup\{-\alpha\}\right) Z\left(A_{s}^{\prime}, B^{\prime}\right) \zeta(1-\alpha-\beta-s) d s .
\end{aligned}
$$

The only differences are that so far we have ignored the arithmetic factors and that in the expression we just derived we have the restrictions $\alpha \in A_{1}$ and $\beta \in B_{1}$. But as $A_{1}$ and $B_{1}$ vary through subsets of $A$ and $B$ every possible $\alpha$ and $\beta$ will appear. Also, we have the terms where $M>N$ and those with $h_{1}=0$.

\section{The same calculation with the arithmetic factors}

We replace $m_{1}$ by $u_{1}$; taking into account the arithmetic considerations and also using $u_{1} \ell N=m_{1} m_{2} \leq X$, we have that our sum is $\sum_{\alpha, \beta} Z\left(\left(A_{1}^{\prime}\right)_{-\alpha}\right) Z\left(\left(B_{1}^{\prime}\right)_{-\beta}\right)$

$$
\begin{aligned}
& \times \sum_{\substack{M \leq N \\
(M, N)=1}} \phi\left(\frac{M}{Q}\right) \phi\left(\frac{N}{Q}\right) \sum_{h_{1}} \sum_{\ell} \frac{\tau_{A_{2}}(N \ell) \tau_{B_{2}}(M \ell)}{M^{1-\beta} N^{1+\beta} \ell} \\
& \times \int_{u_{1} \ell \leq \frac{X}{N}} \sum_{d \mid h_{1}} \sum_{q=1}^{\infty} \frac{\mu(q)(q d, M)^{1-\beta}(q d, N)^{1-\alpha}}{d^{1-\alpha-\beta} q^{2-\alpha-\beta}} \\
& \times G_{A_{1}}\left(1-\alpha, \frac{q d}{(q d, N)}\right) G_{B_{1}}\left(1-\beta, \frac{q d}{(q d, M)}\right) \hat{\psi}\left(\frac{T h_{1}}{2 \pi u_{1} N}\right) \frac{d u_{1}}{u_{1}^{1+\alpha+\beta}} .
\end{aligned}
$$

The term with $h_{1}=0$ just leads to diagonal terms which are easy to deal with. Now we group the non-zero terms $h_{1}$ and $-h_{1}$ together and use $\psi(-v)=\overline{\psi(v)}$. We replace $h_{1}$ by $h_{1} d$. We make the substitution $v_{1}=\frac{T h_{1} d}{2 \pi u_{1} N}$ in the integral and switch the integral over $v_{1}$ with the sum over $h_{1}, d$ and $\ell$. Then (with $h_{1}>0$ ) we have that

$$
\frac{\ell N T h_{1} d}{2 \pi v_{1} N}=u_{1} \ell N \leq X
$$

implies that

$$
\ell h_{1} d \leq \frac{2 \pi X v_{1}}{T} .
$$

Thus we have

$$
\begin{aligned}
& \sum_{\substack{\alpha \in A_{1} \\
\beta \in B_{1}}}\left(\frac{T}{2 \pi}\right)^{-\alpha-\beta} Z\left(\left(A_{1}^{\prime}\right)_{-\alpha}\right) Z\left(\left(B_{1}^{\prime}\right)_{-\beta}\right) \sum_{\substack{M \leq N \\
(M, N)=1}} \frac{\phi\left(\frac{M}{Q}\right) \phi\left(\frac{N}{Q}\right)}{M^{1-\beta} N^{1-\alpha}} \int_{0}^{\infty}\left(2 \Re \hat{\psi}\left(v_{1}\right)\right) \\
& \quad \times \sum_{h_{1} \ell d \leq \frac{2 \pi X_{\nu_{1}}}{T}} \frac{\tau_{A_{2}}(N \ell) \tau_{B_{2}}(M \ell)}{h_{1}^{\alpha+\beta} \ell d} \sum_{q \geq 1} \frac{\mu(q)(q d, M)^{1-\beta}(q d, N)^{1-\alpha}}{q^{2-\alpha-\beta}} \\
& \quad \times G_{A_{1}}\left(1-\alpha, \frac{q d}{(q d, N)}\right) G_{B_{1}}\left(1-\beta, \frac{q d}{(q d, M)}\right) \frac{d \nu_{1}}{v_{1}^{1-\alpha-\beta}} .
\end{aligned}
$$


Now we use Perron's formula to evaluate the sum over $h_{1} \ell d$. This gives

$$
\begin{aligned}
& \sum_{\substack{\alpha \in A_{1} \\
\beta \in B_{1}}}\left(\frac{T}{2 \pi}\right)^{-\alpha-\beta} Z\left(\left(A_{1}^{\prime}\right)_{-\alpha}\right) Z\left(\left(B_{1}^{\prime}\right)_{-\beta}\right) \sum_{\substack{M \leq N \\
(M, N)=1}} \frac{\phi\left(\frac{M}{Q}\right) \phi\left(\frac{N}{Q}\right)}{M^{1-\beta} N^{1-\alpha}} \frac{1}{2 \pi i} \int_{(2)} \\
& \quad \times \int_{0}^{\infty}\left(2 \Re \hat{\psi}\left(v_{1}\right)\right)\left(\frac{2 \pi X v_{1}}{T}\right)^{s} \sum_{h_{1}, \ell, d} \frac{\tau_{A_{2}}(N \ell) \tau_{B_{2}}(M \ell)}{h_{1}^{s+\alpha+\beta} \ell^{1+s} d^{1+s}} \sum_{q \geq 1} \frac{\mu(q)(q d, N)^{1-\alpha}(q d, M)^{1-\beta}}{q^{2-\alpha-\beta}} \\
& \quad \times G_{A_{1}}\left(1-\alpha, \frac{q d}{(q d, N)}\right) G_{B_{1}}\left(1-\beta, \frac{q d}{(q d, M)}\right) \frac{d v_{1}}{v_{1}^{1-\alpha-\beta}} \frac{d s}{s} .
\end{aligned}
$$

The sum over $h_{1}$ is $\zeta(s+\alpha+\beta)$. The integral over $v_{1}$ is

$$
\int_{0}^{\infty} v_{1}^{-1+s+\alpha+\beta}\left(2 \Re \hat{\psi}\left(v_{1}\right)\right) d v_{1}=\chi(1-s-\alpha-\beta) \int_{0}^{\infty} \psi(t) t^{-s-\alpha-\beta} d t .
$$

Combining these two facts and using the functional equation for $\zeta$ we have

$$
\begin{aligned}
& \sum_{\substack{\alpha \in A_{1} \\
\beta \in B_{1}}}\left(\frac{T}{2 \pi}\right)^{-\alpha-\beta} Z\left(\left(A_{1}^{\prime}\right)_{-\alpha}\right) Z\left(\left(B_{1}^{\prime}\right)_{-\beta}\right) \int_{0}^{\infty} t^{-\alpha-\beta} \psi(t) \sum_{\substack{M \leq N \\
(M, N)=1}} \frac{\phi\left(\frac{M}{Q}\right) \phi\left(\frac{N}{Q}\right)}{M^{1-\beta} N^{1-\alpha}} \\
& \quad \times \frac{1}{2 \pi i} \int_{(2)} \zeta(1-s-\alpha-\beta)\left(\frac{2 \pi X}{t T}\right)^{s} \sum_{\ell, d} \frac{\tau_{A_{2}}(N \ell) \tau_{B_{2}}(M \ell)}{\ell^{1+s} d^{1+s}} \\
& \quad \times \sum_{q \geq 1} \frac{\mu(q)(q d, N)^{1-\alpha}(q d, M)^{1-\beta}}{q^{2-\alpha-\beta}} G_{A_{1}}\left(1-\alpha, \frac{q d}{(q d, N)}\right) G_{B_{1}}\left(1-\beta, \frac{q d}{(q d, M)}\right) \frac{d s}{s} d t
\end{aligned}
$$

This requires studying the Dirichlet series

$$
\begin{aligned}
\sum_{(M, N)=1} & \frac{1}{M^{1-\beta} N^{1-\alpha}} \sum_{\ell, d} \frac{\tau_{A_{2}}(N \ell) \tau_{B_{2}}(M \ell)}{\ell^{1+s} d^{1+s}} \sum_{q \geq 1} \frac{\mu(q)(q d, N)^{1-\alpha}(q d, M)^{1-\beta}}{q^{2-\alpha-\beta}} \\
& \times G_{A_{1}}\left(1-\alpha, \frac{q d}{(q d, N)}\right) G_{B_{1}}\left(1-\beta, \frac{q d}{(q d, M)}\right)
\end{aligned}
$$

See the appendix for the resolution of this arithmetic factor.

Regarding multiplicities, see the section on automorphisms at the end of the paper.

Taking account of the terms with $h_{1}=0$ and $h_{2} \neq 0$ we find that we have now accounted for all of the one-swap terms from the semi-diagonal contributions.

\section{$7 h_{1} h_{2} \neq 0$}

Now we come to the crux of the paper, the terms where neither $h_{1}$ nor $h_{2}$ are 0 ; we need to match these up with the two swap terms.

In the formula (3) above we replace the convolution sums by their averages, i.e.

$$
\iint_{u_{1} u_{2} \leq X}\left\langle\tau_{A_{1}}\left(m_{1}\right) \tau_{B_{1}}\left(n_{1}\right)\right\rangle_{m_{1} \sim u_{1}}^{\left.(*)_{1}\right)}\left\langle\tau_{A_{2}}\left(m_{2}\right) \tau_{B_{2}}\left(n_{2}\right)\right\rangle_{m_{2} \sim u_{2}}^{\left(*_{2}\right)} \hat{\psi}\left(\frac{T h_{1}}{2 \pi u_{1} N}+\frac{T h_{2}}{2 \pi u_{2} M}\right) \frac{d u_{1}}{u_{1}} \frac{d u_{2}}{u_{2}}
$$


We insert the formula (4) for these averages. After switching the ensuing sums over $h_{1}, h_{2}$ and $d_{1}, d_{2}$ we have $\sum_{M, N} \phi(M / Q) \phi(N / Q)$ times

$$
\begin{aligned}
& \sum_{\substack{\alpha_{1} \in A_{1} \\
\alpha_{2} \in A_{2}}} \sum_{\beta_{1} \in B_{1} \in B_{2}} Z\left(\left(A_{1}^{\prime}\right)_{-\alpha_{1}}\right) Z\left(\left(A_{2}^{\prime}\right)_{-\alpha_{2}}\right) Z\left(\left(B_{1}^{\prime}\right)_{-\beta_{1}}\right) Z\left(\left(B_{2}^{\prime}\right)_{-\beta_{2}}\right) \sum_{\substack{q_{1}, d_{1}, h_{1} \\
q_{2}, d_{2}, h_{2}}} \frac{\mu\left(q_{1}\right) \mu\left(q_{2}\right)}{q_{1}^{2-\alpha_{1}-\beta_{1}} q_{2}^{2-\alpha_{2}-\beta_{2}}} \\
& \quad \times \frac{G_{A_{1}}\left(1-\alpha_{1}, \frac{q_{1} d_{1}}{\left(q_{1} d_{1}, N\right)}\right) G_{A_{2}}\left(1-\alpha_{2}, \frac{q_{2} d_{2}}{\left(q_{2} d_{2}, M\right)}\right) G_{B_{1}}\left(1-\beta_{1}, \frac{q_{1} d_{1}}{\left(q_{1} d_{1}, M\right)}\right) G_{B_{2}}\left(1-\beta_{2}, \frac{q_{2} d_{2}}{\left(q_{2} d_{2}, N\right)}\right)}{\left(q_{1} d_{1}, N\right)^{-1+\alpha_{1}}\left(q_{1} d_{1}, M\right)^{-1+\beta_{1}}\left(q_{2} d_{2}, M\right)^{-1+\alpha_{2}}\left(q_{2} d_{2}, N\right)^{-1+\beta_{2}} d_{1}^{1-\alpha_{1}-\beta_{1}} d_{2}^{1-\alpha_{2}-\beta_{2}}} \\
& \quad \times \iint_{T^{2} \leq u_{1} u_{2} \leq X} M^{-1+\beta_{1}-\beta_{2}} N^{-1+\beta_{2}-\beta_{1}} u_{1}^{-\alpha_{1}-\beta_{1}} u_{2}^{-\alpha_{2}-\beta_{2}} \\
& \quad \times \hat{\psi}\left(\frac{T h_{1} d_{1}}{2 \pi u_{1} N}+\frac{T h_{2} d_{2}}{2 \pi u_{2} M}\right) \frac{d u_{1}}{u_{1}} \frac{d u_{2}}{u_{2}} .
\end{aligned}
$$

Let's first assume that $h_{1}>0$ and $h_{2}>0$. We make the changes of variable $\nu_{1}=\frac{T h_{1} d_{1}}{2 \pi u_{1} N}$ and $v_{2}=\frac{T h_{2} d_{2}}{2 \pi u_{2} M}$ and bring the sums over $h_{1}$ and $h_{2}$ to the inside; $u_{1} u_{2}<X$ implies that

$$
h_{1} d_{1} h_{2} d_{2}<\frac{4 \pi^{2} X v_{1} v_{2} M N}{T^{2}} .
$$

Then the sums over the $q_{i}, h_{i}, d_{i}$ are $N^{-1+\alpha_{1}+\beta_{2}} M^{-1+\alpha_{2}+\beta_{1}}$ times

$$
\begin{aligned}
& \left(\frac{T}{2 \pi}\right)^{-\alpha_{1}-\alpha_{2}-\beta_{1}-\beta_{2}} \iint_{v_{1}, v_{2}} v_{1}^{\alpha_{1}+\beta_{1}} v_{2}^{\alpha_{2}+\beta_{2}} \hat{\psi}\left(v_{1}+v_{2}\right) \frac{1}{2 \pi i} \int_{(2)} \sum_{\substack{q_{1}, d_{1}, h_{1} \\
q_{2}, d_{2}, h_{2}}} \frac{\mu\left(q_{1}\right) \mu\left(q_{2}\right)}{q_{1}^{2-\alpha_{1}-\beta_{1}} q_{2}^{2-\alpha_{2}-\beta_{2}}} \\
& \times \frac{G_{A_{1}}\left(1-\alpha_{1}, \frac{q_{1} d_{1}}{\left(q_{1} d_{1}, N\right)}\right) G_{A_{2}}\left(1-\alpha_{2}, \frac{q_{2} d_{2}}{\left(q_{2} d_{2}, M\right)}\right) G_{B_{1}}\left(1-\beta_{1}, \frac{q_{1} d_{1}}{\left(q_{1} d_{1}, M\right)}\right) G_{B_{2}}\left(1-\beta_{2}, \frac{q_{2} d_{2}}{\left(q_{2} d_{2}, N\right)}\right)}{\left(q_{1} d_{1}, N\right)^{-1+\alpha_{1}}\left(q_{1} d_{1}, M\right)^{-1+\beta_{1}}\left(q_{2} d_{2}, M\right)^{-1+\alpha_{2}}\left(q_{2} d_{2}, N\right)^{-1+\beta_{2}} d_{1}^{1+s} d_{2}^{1+s} h_{1}^{\alpha_{1}+\beta_{1}+s} h_{2}^{\alpha_{2}+\beta_{2}+s}} \\
& \times \frac{\left(\frac{4 \pi^{2} X v_{1} v_{2} M N}{T^{2}}\right)^{s}}{s} d s \frac{d v_{1}}{v_{1}} \frac{d v_{2}}{v_{2}} .
\end{aligned}
$$

The sums over $h_{1}$ and $h_{2}$ are $\zeta\left(s+\alpha_{1}+\beta_{1}\right) \zeta\left(s+\alpha_{2}+\beta_{2}\right)$. The other 3 cases of the signs of $h_{1}$ and $h_{2}$ can be taken care of similarly. Then we use

$$
\hat{\psi}\left(v_{1}+v_{2}\right)=\int_{0}^{\infty} \psi(t) e\left(t\left(v_{1}+v_{2}\right)\right) d t
$$

to see that

$$
\begin{gathered}
\hat{\psi}\left(v_{1}+v_{2}\right)+\hat{\psi}\left(v_{1}-v_{2}\right)+\hat{\psi}\left(-v_{1}+v_{2}\right)+\hat{\psi}\left(-v_{1}-v_{2}\right) \\
=\int_{0}^{\infty} \psi(t)\left(e\left(t v_{1}\right)+e\left(-t v_{1}\right)\right)\left(e\left(t v_{2}\right)+e\left(-t v_{2}\right)\right) d t .
\end{gathered}
$$

Also

$$
\int_{0}^{\infty} v_{1}^{s+\alpha+\gamma-1}\left(e\left(t v_{1}\right)+e\left(-t v_{1}\right)\right) d v_{1}=t^{-s-\alpha-\gamma} \chi(1-s-\alpha-\gamma),
$$

and similarly for the integral over $v_{2}$. This leaves us with a total for the sum over $M, N, q_{i}, h_{i}, d_{i}$ of

$$
\begin{aligned}
& \int_{0}^{\infty} \psi(t)\left(\frac{t T}{2 \pi}\right)^{-\alpha_{1}-\alpha_{2}-\beta_{1}-\beta_{2}} \frac{1}{2 \pi i} \int_{(2)} \frac{\left(\frac{4 \pi^{2} X}{t^{2} T^{2}}\right)^{s}}{s} \zeta\left(1-s-\alpha_{1}-\beta_{1}\right) \zeta\left(1-s-\alpha_{2}-\beta_{2}\right) \\
& \times \sum_{\substack{(M, N)=1 \\
M \leq N}} \frac{\phi(M / Q) \phi(N / Q)}{M^{1-s-\alpha_{2}-\beta_{1}} N^{1-s-\alpha_{1}-\beta_{2}}} \sum_{\substack{q_{1}, d_{1} \\
q_{2}, d_{2}}} \frac{\mu\left(q_{1}\right) \mu\left(q_{2}\right)}{q_{1}^{2-\alpha_{1}-\beta_{1}} q_{2}^{2-\alpha_{2}-\beta_{2}}} \frac{G_{A_{1}}\left(1-\alpha_{1}, \frac{q_{1} d_{1}}{\left(q_{1} d_{1}, N\right)}\right)}{\left(q_{1} d_{1}, N\right)^{-1+\alpha_{1}}} \\
& \times \frac{G_{A_{2}}\left(1-\alpha_{2}, \frac{q_{2} d_{2}}{\left(q_{2} d_{2}, M\right)}\right) G_{B_{1}}\left(1-\beta_{1}, \frac{q_{1} d_{1}}{\left(q_{1} d_{1}, M\right)}\right) G_{B_{2}}\left(1-\beta_{2}, \frac{q_{2} d_{2}}{\left(q_{2} d_{2}, N\right)}\right)}{\left(q_{1} d_{1}, M\right)^{-1+\beta_{1}}\left(q_{2} d_{2}, M\right)^{-1+\alpha_{2}}\left(q_{2} d_{2}, N\right)^{-1+\beta_{2}} d_{1}^{1+s} d_{2}^{1+s}} d s d t .
\end{aligned}
$$


Recall that

$$
G_{A}(1-\alpha, p)=\tau_{A^{\prime}}(p)+O(1 / p)
$$

we use this to calculate the polar part of

$$
\sum_{\substack{d_{1}, d_{2} \\(M, N)=1 \\ M \leq N}} \frac{\tau_{A_{1}^{\prime}}\left(\frac{d_{1}}{\left(d_{1}, N\right)}\right) \tau_{B_{1}^{\prime}}\left(\frac{d_{1}}{\left(d_{1}, M\right)}\right) \tau_{A_{2}^{\prime}}\left(\frac{d_{2}}{\left(d_{2}, M\right)}\right) \tau_{B_{2}^{\prime}}\left(\frac{d_{2}}{\left(d_{2}, N\right)}\right)}{\left(d_{1}, N\right)^{-1+\alpha_{1}}\left(d_{1}, M\right)^{-1+\beta_{1}}\left(d_{2}, M\right)^{-1+\alpha_{2}}\left(d_{2}, N\right)^{-1+\beta_{2}} d_{1}^{1+s} d_{2}^{1+s} M^{1-s-\alpha_{2}-\beta_{1}} N^{1-s-\alpha_{1}-\beta_{2}}} .
$$

We do this by calculating the significant parts of the Euler product. The following table is helpful; we let $A_{1}^{\prime}=A_{1}-\left\{\alpha_{1}\right\}, A_{2}^{\prime}=A_{2}-\left\{\alpha_{2}\right\}, B_{1}^{\prime}=B_{1}-\left\{\beta_{1}\right\}$, and $B_{2}^{\prime}=B_{2}-\left\{\beta_{2}\right\}$.

\begin{tabular}{|l|l|l|l|l|l|}
\hline$d_{1}$ & $d_{2}$ & $N$ & $M$ & Euler term & $Z$ - factor \\
\hline$p$ & 1 & 1 & 1 & $\tau_{A_{1}^{\prime}}(p) \tau_{B_{1}^{\prime}}(p) / p^{1+s}$ & $Z\left(\left(A_{1}^{\prime}\right)_{s}, B_{1}^{\prime}\right)$ \\
\hline 1 & $p$ & 1 & 1 & $\tau_{A_{2}^{\prime}}(p) \tau_{B_{2}^{\prime}}(p) / p^{1+s}$ & $Z\left(\left(A_{2}^{\prime}\right)_{s}, B_{2}^{\prime}\right)$ \\
\hline 1 & 1 & $p$ & 1 & $p^{\alpha_{1}+\beta_{2}} / p^{1-s}$ & $Z\left(\left\{-\alpha_{1}-s\right\},\left\{-\beta_{2}\right\}\right)$ \\
\hline 1 & 1 & 1 & $p$ & $p^{\alpha_{2}+\beta_{1}} / p^{1-s}$ & $Z\left(\left\{-\alpha_{2}-s\right\},\left\{-\beta_{1}\right\}\right)$ \\
\hline$p$ & 1 & $p$ & 1 & $\tau_{B_{1}^{\prime}}(p) / p^{1-\beta_{2}}$ & $Z\left(B_{1}^{\prime},\left\{-\beta_{2}\right\}\right)$ \\
\hline$p$ & 1 & 1 & $p$ & $\tau_{A_{1}^{\prime}}(p) / p^{1-\alpha_{2}}$ & $Z\left(A_{1}^{\prime},\left\{-\alpha_{2}\right\}\right)$ \\
\hline 1 & $p$ & $p$ & 1 & $\tau_{A_{2}^{\prime}}(p) / p^{1-\alpha_{1}}$ & $Z\left(A_{2}^{\prime},\left\{-\alpha_{1}\right\}\right)$ \\
\hline 1 & $p$ & 1 & $p$ & $\tau_{B_{2}^{\prime}}(p) / p^{1-\beta_{1}}$ & $Z\left(B_{2}^{\prime},\left\{-\beta_{1}\right\}\right)$ \\
\hline$p$ & $p$ & $p$ & 1 & $\tau_{A_{2}^{\prime}}(p) \tau_{B_{1}^{\prime}}(p) / p^{1+s}$ & $Z\left(\left(A_{2}^{\prime}\right)_{s}, B_{1}^{\prime}\right)$ \\
\hline$p$ & $p$ & 1 & $p$ & $\tau_{A_{1}^{\prime}}(p) \tau_{B_{2}^{\prime}}(p) / p^{1+s}$ & $Z\left(\left(A_{1}^{\prime}\right)_{s}, B_{2}^{\prime}\right)$ \\
\hline
\end{tabular}

If we include the factors $Z\left(\left(A_{1}^{\prime}\right)_{s},\left\{-s-\alpha_{1}\right\}\right) Z\left(B_{1}^{\prime},\left\{-\beta_{1}\right\}\right), Z\left(\left(A_{2}^{\prime}\right)_{s},\left\{-s-\alpha_{2}\right\}\right) Z\left(B_{2}^{\prime},\left\{-\beta_{2}\right\}\right)$, $Z\left(\left\{-s-\alpha_{1}\right\},\left\{-\beta_{1}\right\}\right)$ and $Z\left(\left\{-s-\alpha_{2}\right\},\left\{-\beta_{2}\right\}\right)$ then the product of all of these $Z$-factors is

$$
\begin{aligned}
& Z\left(\left(A_{1}^{\prime} \cup A_{2}^{\prime}\right)_{s} \cup\left\{-\beta_{1}\right\} \cup\left\{-\beta_{2}\right\}, B_{1}^{\prime} \cup B_{2}^{\prime} \cup\left\{-s-\alpha_{1}\right\} \cup\left\{-s-\alpha_{2}\right\}\right) \\
& =Z\left((A-S)_{s}+T^{-}, B-T+\left(S_{s}\right)^{-}\right)
\end{aligned}
$$

where $S=\left\{\alpha_{1}, \alpha_{2}\right\}$ and $T=\left\{\beta_{1}, \beta_{2}\right\}$.

The predicted two-swap terms from the recipe are

$$
\begin{aligned}
& \sum_{\substack{S \subset A, T \subset B \\
|S|=|T|=2}} \int_{0}^{\infty} \psi(t)\left(\frac{t T}{2 \pi}\right){ }^{-\sum_{\alpha \in S}(\alpha+\beta)} \\
& \quad \times \frac{1}{2 \pi i} \int_{(2)} \frac{\left(\frac{4 \pi^{2} X}{t^{2} T^{2}}\right)^{s}}{s} \mathcal{A} Z\left((A-S)_{s}+T^{-}, B-T+S_{s}^{-}\right) d s d t
\end{aligned}
$$

which matches the above except that $S$ and $T$ are allowed to range over all two-element subsets of $A$ and $B$ in the recipe version whereas in the correlation version we first split $A=A_{1} \cup A_{2}$ and $B=B_{1} \cup B_{2}$ and then take one element from $A_{1}$ and one from $A_{2}$ to make up our two element set $S$ and similarly one element from $B_{1}$ and one from $B_{2}$ to make our two element set $T$.

See the last two sections for the calculation of the arithmetic factor.

\section{Automorphisms}

The final step of this paper is to explain the apparent over-counting that has occurred. The explanation is that there are automorphisms that have to be taken into account. In this section we explain these multiplicities. 
We start with

$$
\left\{\begin{array}{l}
N m_{1}=M n_{1}+h_{1} \\
M m_{2}=N n_{2}+h_{2}
\end{array}\right.
$$

Suppose $m_{1}=\mu_{1} \hat{\mu_{1}}, m_{2}=\mu_{2} \hat{\mu_{2}}, n_{1}=v_{1} \hat{v_{1}}$ and $n_{2}=v_{2} \hat{v_{2}}$. Multiply the first equation by $\mu_{2} \nu_{2}$ and the second equation by $\mu_{1} v_{1}$. Let

$$
\tilde{M}=v_{1} \mu_{2} M ; \quad \tilde{N}=\mu_{1} v_{2} N ; \quad \tilde{m_{1}}=\hat{\mu_{1}} \mu_{2} ; \quad \tilde{m_{2}}=\mu_{1} \hat{\mu_{2}} ; \quad \tilde{n_{1}}=\hat{v_{1}} v_{2} ; \quad \tilde{n_{2}}=v_{1} \hat{v_{2}} .
$$

Then we have

$$
\left\{\begin{array}{l}
\tilde{N} \tilde{n}_{1}=\tilde{M} \tilde{n}_{1}+\tilde{h_{1}} \\
\tilde{M} \tilde{m}_{2}=\tilde{N} \tilde{n_{2}}+\tilde{h_{2}}
\end{array}\right.
$$

where

$$
\tilde{h_{1}}=\mu_{2} v_{2} h_{1} \quad \text { and } \quad \tilde{h_{2}}=\mu_{1} v_{1} h_{2} .
$$

This scheme provides lots of automorphisms and explains the overcounting we have.

Basically there is one automorphism for each quadruple of divisors of $m_{1}, m_{2}, n_{1}$ and $n_{2}$. We have $m=m_{1} m_{2}$ and $n=n_{1} n_{2}$ where if $|A|=k$ and $|B|=\ell$ then $\tau_{A}$ is a convolution of $k$ and $\tau_{B}$ a convolution of $\ell$ atomic functions. We can think of

$$
A=\left\{\alpha_{1}, \ldots, \alpha_{k}\right\} \quad B=\left\{\beta_{1}, \ldots, \beta_{\ell}\right\}
$$

and with $I=\{1,2, \ldots, k\}$ and $J=\{1,2, \ldots, \ell\}$ we partition $I=I_{1} \cup I_{2}$ and $J=J_{1} \cup J_{2}$. Then in our decompositions $A=A_{1} \cup A_{2}$ and $B=B_{1} \cup B_{2}$ we have $A_{1}=\left\{\alpha_{i}: i \in I_{1}\right\}$ etc. These correspond to the decompositions $m=m_{1} m_{2}$ and $n=n_{1} n_{2}$. If we write $m=\mu_{1} \ldots \mu_{k}$ and $n=v_{1} \ldots v_{\ell}$ then we can put $m_{1}=\prod_{i \in I_{1}} \mu_{i}$ etc. The number of such decompositions of $A$ or of $m$ is just the number of subsets of $A$, i.e. $2^{k}$; and the number for $B$ is $2^{\ell}$. We can associate an automorphism as above with each such decomposition. Therefore, there are $2^{k+\ell}$ automorphisms in total. So each term is counted with a multiplicity $2^{k+\ell}$. Now let's see that this overcounting is in agreement with the number of ways of producing the term from the recipe with, say, $S=\left\{\alpha_{1}, \alpha_{2}\right\}$ and $T=\left\{\beta_{1}, \beta_{2}\right\}$. The term from the recipe will occur whenever we have a decomposition of $A=A_{1} \cup A_{2}$ and $B=B_{1} \cup B_{2}$ in which precisely one of $\alpha_{1}$ and $\alpha_{2}$ is in $A_{1}$ and the other in $A_{2}$ and similarly for $B$ and the $\beta \mathrm{s}$. How many ways are there to do this? If we say that $\alpha_{1}$ is to be in $A_{1}$ and $\alpha_{2}$ in $A_{2}$ then we have $k-2$ other elements to be partitioned into two sets. There are $2^{k-2}$ subsets and the chosen subset can be assigned to go with $\alpha_{1}$ or with $\alpha_{2}$, so we have an extra factor of 2; then and then another factor of 2 by putting $\alpha_{1}$ in $A_{2}$ and $\alpha_{2}$ in $A_{1}$. Therefore, a total of $2^{k}$ ways to do this. And $2^{\ell}$ for the $\beta$ s into the $B$ s. So, we have the same amount of overcounting as there are automorphisms. Taking this into account, we obtain just a single copy of each term from the recipe.

\section{Conclusion}

We have shown how to obtain an asymptotic formula with power savings for the mean square of a Dirichlet polynomial of length $X$ where $T^{2} \ll X \ll T^{3}$ with coefficients that are general divisor functions in two different ways: one way is via Perron's formula and the recipe, and the other is by calculating a convolution of shifted divisor correlations. The two approaches give exactly the same answer.

In the next paper, which will conclude this introductory series, we will consider the completely general situation with an arbitrary length Dirichlet polynomial. 


\section{The semi-diagonal arithmetic factor}

It remains to prove that the arithmetic factors agree. This calculation is surprisingly involved. In order to carry it out with minimal notational difficulties we introduce a new set of notation. These appendices are self-contained.

We begin by introducing a little notation. First of all, we are working locally; basically we are identifying the local $p$-factor in an Euler product. As far as we are concerned $p$ is fixed for this discussion so we often suppress it. In fact we write $X$ for $1 / p$ and mostly consider power series in $X$. We take the unusual step of suppressing not only the prime $p$ but the divisor function and so we write $A(n)$ in place of $\tau_{A}\left(p^{n}\right)$. Also, for a set $A$ we let

$$
A_{\alpha}=\{a+\alpha: a \in A\} .
$$

A further piece of notation: $A^{+}=A \cup\{0\}$. We have two important identities. The first is

$$
A^{+}(d)=A(d)+A^{+}(d-1) .
$$

This is a special case of

$$
(A \cup\{-\alpha\})(d-1)=X^{\alpha}((A \cup\{-\alpha\})(d)-A(d)) .
$$

The other identity is

$$
\sum_{r=0}^{R} A(r+M)=A^{+}(R+M)-A^{+}(M-1)
$$

which follows by repeated application of the first identity.

For arbitrary sets $A, B, C$ and $D$ we let

$$
\mathcal{C}(A, B):=\sum_{M=0}^{\infty} A(M) B(M) X^{M}
$$

and

$$
\mathcal{F}(A, B ; C, D)=\sum_{K, L, M} A(K) B(K+M) C(L) D(L+M) X^{K+L+M} ;
$$

Also, we let

$$
Z(A)=\sum_{j=0}^{\infty} A(j) X^{j}=\prod_{a \in A}\left(1-X^{1+a}\right)^{-1} .
$$

We have a lemma about $\mathcal{F}$ and $\mathcal{C}$ which is really just a formal manipulation; consequently we state it in a more general form.

Lemma 1 For any 4 functions $a, A, b$, B let

$$
F(a, A ; b, B)=\sum_{K, L, M} a(K) A(K+M) b(L) B(L+M) X^{K+L+M}
$$

and

$$
C(a, b)=\sum_{r=0}^{\infty} a(r) b(r) X^{r}
$$

we have

$$
F(a, A ; b, B)+F(A, a ; B, b)=C(A \star b, a \star B)+C(a, A) C(b, B) .
$$


Proof Let $Y=\sqrt{X} e(\theta)$. Then

$$
\begin{aligned}
F(a, A ; b, B) & =\int_{0}^{1} \sum_{r, s, M, N} a(r) A(r+M) Y^{r+s+M} b(s) B(s+N) \bar{Y}^{r+s+N} d \theta \\
& =\int_{0}^{1} \sum_{\substack{R, S \\
r \leq R ; s \leq S}} a(r) A(R) Y^{s+R} b(s) B(S) \bar{Y}^{r+S} d \theta \\
& =\sum_{\substack{r+S=R+s \\
r \leq R ; s \leq S}} a(r) A(R) b(s) B(S) X^{r+S}
\end{aligned}
$$

The latter sum is

$$
\begin{gathered}
\sum_{r+S=R+s} a(r) A(R) b(s) B(S) X^{r+S}-\sum_{\substack{r+S=R+s \\
r>R ; s>S}} a(r) A(R) b(s) B(S) X^{r+S} \\
=C(a \star B, A \star b)+C(a, A) C(b, B)-F(A, a ; B, b)
\end{gathered}
$$

as desired.

Now we address the arithmetic factor from the semi-diagonal term. The $p$ part of

$$
\begin{aligned}
& Z\left(\left(A_{1}^{\prime}\right)_{-\alpha}\right) Z\left(\left(B_{1}^{\prime}\right)_{-\beta}\right) \sum_{(M, N)=1} \frac{1}{M^{1-\beta} N^{1-\alpha}} \sum_{\ell, d} \frac{\tau_{A_{2}}(N \ell) \tau_{B_{2}}(M \ell)}{\ell^{1+s} d^{1+s}} \\
& \quad \times \sum_{q \geq 1} \frac{\mu(q)(q d, N)^{1-\alpha}(q d, M)^{1-\beta}}{q^{2-\alpha-\beta}} \\
& \quad \times G_{A_{1}}\left(1-\alpha, \frac{q d}{(q d, N)}\right) G_{B_{1}}\left(1-\beta, \frac{q d}{(q d, M)}\right) s
\end{aligned}
$$

is (after setting $s=0$ )

$$
\begin{aligned}
& \quad \sum_{\min (M, N)=0} X^{M(1-\beta)+N(1-\alpha)} \sum_{\ell, d} A_{2}(N+\ell) B_{2}(M+\ell) X^{\ell+d} \\
& \times \sum_{q} \mu\left(p^{q}\right) X^{-\min (q+d, N)(1-\alpha)-\min (q+d, M)(1-\beta)+q(2-\alpha-\beta)} \\
& \times \sum_{j, k} A_{1}^{\prime}(j+q+d-\min (q+d, N)) \\
& \times B_{1}^{\prime}(k+q+d-\min (q+d, M)) X^{j(1-\alpha)+k(1-\beta)}
\end{aligned}
$$

We use

$$
\sum_{\min (M, N)=0} f(M, N)=\sum_{M} f(M, 0)+\sum_{N} f(0, N)-f(0,0)
$$

and get $S_{L}+S_{R}-S_{0}$ where

$$
\begin{aligned}
S_{0}= & \sum_{\ell, d, q, j, k} A_{2}(\ell) B_{2}(\ell) \mu\left(p^{q}\right) A_{1}^{\prime}(j+q+d) B_{1}^{\prime}(k+q+d) \\
& \times X^{q(2-\alpha-\beta)+\ell+d+j(1-\alpha)+k(1-\beta)}, \\
S_{L}= & \sum_{M} X^{M(1-\beta)} \sum_{\ell, d} A_{2}(\ell) B_{2}(M+\ell) X^{\ell+d} \sum_{q} \mu\left(p^{q}\right) X^{-\min (q+d, M)(1-\beta)+q(2-\alpha-\beta)} \\
& \times \sum_{j, k} A_{1}^{\prime}(j+q+d) B_{1}^{\prime}(k+q+d-\min (q+d, M)) X^{j(1-\alpha)+k(1-\beta)}
\end{aligned}
$$




$$
\begin{aligned}
S_{R}= & \sum_{N} X^{N(1-\alpha)} \sum_{\ell, d} A_{2}(N+\ell) B_{2}(\ell) X^{\ell+d} \sum_{q} \mu\left(p^{q}\right) X^{-\min (q+d, N)(1-\alpha)+q(2-\alpha-\beta)} \\
& \times \sum_{j, k} A_{1}^{\prime}(j+q+d-\min (q+d, N)) B_{1}^{\prime}(k+q+d) X^{j(1-\alpha)+k(1-\beta)} .
\end{aligned}
$$

We expand the $q$ sum in $S_{0}$ to get

$$
\begin{aligned}
S_{0}= & \sum_{\ell, d, j, k} A_{2}(\ell) B_{2}(\ell) A_{1}^{\prime}(j+d) B_{1}^{\prime}(k+d) X^{\ell+d+j(1-\alpha)+k(1-\beta)} \\
& -\sum_{\ell, d, j, k} A_{2}(\ell) B_{2}(\ell) A_{1}^{\prime}(j+1+d) B_{1}^{\prime}(k+1+d) X^{2-\alpha-\beta+\ell+d+j(1-\alpha)+k(1-\beta)} .
\end{aligned}
$$

This telescopes in $j$ and $k$ to give

$$
\begin{aligned}
S_{0}= & \sum_{\ell, d, j} A_{2}(\ell) B_{2}(\ell) A_{1}^{\prime}(j+d) B_{1}^{\prime}(d) X^{\ell+d+j(1-\alpha)} \\
& +\sum_{\ell, d, k} A_{2}(\ell) B_{2}(\ell) A_{1}^{\prime}(d) B_{1}^{\prime}(k+d) X^{\ell+d+k(1-\beta)} \\
& -\sum_{\ell, d} A_{2}(\ell) B_{2}(\ell) A_{1}^{\prime}(d) B_{1}^{\prime}(d) X^{\ell+d} \\
= & \mathcal{C}\left(A_{2}, B_{2}\right)\left(\sum_{r} X^{r(1-\alpha)} A_{1}^{\prime}(r) \sum_{d \leq r} X^{d \alpha} B_{1}^{\prime}(d)\right. \\
& \left.+\sum_{r} X^{r(1-\beta)} B_{1}^{\prime}(r) \sum_{d \leq r} X^{d \beta} A_{1}^{\prime}(d)-\mathcal{C}\left(A_{1}^{\prime}, B_{1}^{\prime}\right)\right) \\
= & \mathcal{C}\left(A_{2}, B_{2}\right)\left(\sum_{r} X^{r(1-\alpha)} A_{1}^{\prime}(r)\left(\left(B_{1}^{\prime}\right)_{\alpha}\right)^{+}(r)\right. \\
& \left.+\sum_{r} X^{r(1-\beta)} B_{1}^{\prime}(r)\left(\left(A_{1}^{\prime}\right)_{\beta}\right)^{+}(r)-\mathcal{C}\left(A_{1}^{\prime}, B_{1}^{\prime}\right)\right) \\
= & \mathcal{C}\left(A_{2}, B_{2}\right)\left(\mathcal{C}\left(\left(A_{1}^{\prime}\right)_{-\alpha},\left(\left(B_{1}^{\prime}\right)_{\alpha}\right)^{+}\right)+\mathcal{C}\left(\left(B_{1}^{\prime}\right)_{-\beta},\left(\left(A_{1}^{\prime}\right)_{\beta}\right)^{+}\right)-\mathcal{C}\left(A_{1}^{\prime}, B_{1}^{\prime}\right)\right)
\end{aligned}
$$

This may be rewritten as

$$
S_{0}=\mathcal{C}\left(A_{2}, B_{2}\right)\left(\mathcal{C}\left(A_{1}^{\prime}, B_{1}^{\prime} \cup\{-\alpha\}\right)+\mathcal{C}\left(B_{1}^{\prime}, A_{1}^{\prime} \cup\{-\beta\}\right)-\mathcal{C}\left(A_{1}^{\prime}, B_{1}^{\prime}\right)\right) .
$$

Now we turn to $S_{L}$. Expanding in $q$ we have

$$
\begin{aligned}
S_{L}= & \sum_{M, \ell, d, j, k} A_{2}(\ell) B_{2}(M+\ell) A_{1}^{\prime}(j+d) B_{1}^{\prime}(k+d-\min (d, M)) \\
& \times X^{\ell+d+M(1-\beta)-\min (d, M)(1-\beta)+j(1-\alpha)+k(1-\beta)} \\
& -\sum_{M, \ell, d, j, k} A_{2}(\ell) B_{2}(M+\ell) A_{1}^{\prime}(j+1+d) B_{1}^{\prime}(k+1+d-\min (1+d, M)) \\
& \times X^{\ell+d+M(1-\beta)-\min (1+d, M)(1-\beta)+2-\alpha-\beta+j(1-\alpha)+k(1-\beta)} .
\end{aligned}
$$

We split this into $S_{L}=S_{L}^{-}+S_{L}^{+}$where $S_{L}^{-}$denotes those terms for which $d<M$ and $S_{L}^{+}$ contains those terms with $d \geq M$. We have

$$
\begin{aligned}
S_{L}^{-}= & \sum_{\substack{M, \ell, j, j, k \\
d<M}} A_{2}(\ell) B_{2}(M+\ell) A_{1}^{\prime}(j+d) B_{1}^{\prime}(k) X^{\ell+M(1-\beta)+d \beta+j(1-\alpha)+k(1-\beta)} \\
& -\sum_{\substack{M, \ell, j, j, k \\
d<M}} A_{2}(\ell) B_{2}(M+\ell) A_{1}^{\prime}(j+1+d) B_{1}^{\prime}(k) X^{\ell+M(1-\beta)+d \beta+(j+1)(1-\alpha)+k(1-\beta)} .
\end{aligned}
$$


The sum over $j$ telescopes; we are left with

$$
S_{L}^{-}=\sum_{\substack{M, \ell, k \\ d<M}} A_{2}(\ell) B_{2}(M+\ell) A_{1}^{\prime}(d) B_{1}^{\prime}(k) X^{\ell+M(1-\beta)+d \beta+k(1-\beta)}
$$

We execute the sum over $k$ to obtain

$$
S_{L}^{-}=Z\left(\left(B_{1}^{\prime}\right)_{-\beta}\right) \sum_{\substack{M, \ell, d<M}} A_{2}(\ell) B_{2}(M+\ell) A_{1}^{\prime}(d) X^{\ell+M(1-\beta)+d \beta} .
$$

The sum over $d$ gives

$$
\begin{aligned}
S_{L}^{-} & =Z\left(\left(B_{1}^{\prime}\right)_{-\beta}\right) \sum_{M, \ell} A_{2}(\ell) B_{2}(M+\ell)\left(\left(A_{1}^{\prime}\right)_{\beta}\right)^{+}(M-1) X^{\ell+M(1-\beta)} \\
& =Z\left(\left(B_{1}^{\prime}\right)_{-\beta}\right) \sum_{M, \ell} A_{2}(\ell) B_{2}(M+\ell)\left(\left(\left(A_{1}^{\prime}\right)_{\beta}\right)^{+}(M)-\left(A_{1}^{\prime}\right)_{\beta}(M)\right) X^{\ell+M(1-\beta)} \\
& =Z\left(\left(B_{1}^{\prime}\right)_{-\beta}\right) \sum_{M, \ell} A_{2}(\ell) B_{2}(M+\ell)\left(\left(A_{1}^{\prime} \cup\{-\beta\}\right)(M)-A_{1}^{\prime}(M)\right) X^{\ell+M} \\
& =Z\left(\left(B_{1}^{\prime}\right)_{-\beta}\right)\left(\mathcal{C}\left(A_{1}^{\prime} \cup A_{2} \cup\{-\beta\}, B_{2}\right)-\mathcal{C}\left(A_{1}^{\prime} \cup A_{2}, B_{2}\right)\right) .
\end{aligned}
$$

Now we consider $S_{L}^{+}$. We have

$$
\begin{aligned}
S_{L}^{+}= & \sum_{\substack{M, \ell, j, k \\
d \geq M}} A_{2}(\ell) B_{2}(M+\ell) A_{1}^{\prime}(j+d) B_{1}^{\prime}(k+d-M) X^{\ell+d+j(1-\alpha)+k(1-\beta)} \\
& -\sum_{\substack{M, \ell, j, k \\
d \geq M}} A_{2}(\ell) B_{2}(M+\ell) A_{1}^{\prime}(j+1+d) \\
& \times B_{1}^{\prime}(k+1+d-M) X^{\ell+d+2-\alpha-\beta+j(1-\alpha)+k(1-\beta)}
\end{aligned}
$$

This sum telescopes in $j$ and $k$. We have

$$
\begin{aligned}
S_{L}^{+}= & \sum_{\substack{M, \ell, j \\
d \geq M}} A_{2}(\ell) B_{2}(M+\ell) A_{1}^{\prime}(j+d) B_{1}^{\prime}(d-M) X^{\ell+d+j(1-\alpha)} \\
& +\sum_{\substack{M, \ell, k \\
d \geq M}} A_{2}(\ell) B_{2}(M+\ell) A_{1}^{\prime}(d) B_{1}^{\prime}(k+d-M) X^{\ell+d+k(1-\beta)} \\
& -\sum_{\substack{M, \ell \\
d \geq M}} A_{2}(\ell) B_{2}(M+\ell) A_{1}^{\prime}(d) B_{1}^{\prime}(d-M) X^{\ell+d} .
\end{aligned}
$$

We replace $d$ by $d+M$ and have

$$
\begin{aligned}
S_{L}^{+}= & \sum_{M, \ell, j, d} A_{2}(\ell) B_{2}(M+\ell) A_{1}^{\prime}(j+d+M) B_{1}^{\prime}(d) X^{\ell+d+M+j(1-\alpha)} \\
& +\sum_{M, \ell, k, d} A_{2}(\ell) B_{2}(M+\ell) A_{1}^{\prime}(d+M) B_{1}^{\prime}(k+d) X^{\ell+d+M+k(1-\beta)} \\
& -\sum_{M, \ell, d} A_{2}(\ell) B_{2}(M+\ell) A_{1}^{\prime}(d+M) B_{1}^{\prime}(d) X^{\ell+d+M} .
\end{aligned}
$$

In the first term we replace $j+d$ by $r$ and sum over $d$; it becomes

$$
\sum_{M, \ell, r} A_{2}(\ell) B_{2}(M+\ell)\left(A_{1}^{\prime}\right)_{-\alpha}(r+M)\left(\left(B_{1}^{\prime}\right)_{\alpha}\right)^{+}(r) X^{\ell+r+M+M \alpha} .
$$


In the second term we execute the sum over $k$ as follows:

$$
\begin{aligned}
& \sum_{k, d} A_{1}^{\prime}(d+M) B_{1}^{\prime}(k+d) X^{d+k(1-\beta)} \\
& \quad=\sum_{K}\left(B_{1}^{\prime}\right)_{-\beta}(K) \sum_{d \leq K} A_{1}^{\prime}(d+M) X^{d+K \beta} \\
& =X^{-M \beta} \sum_{K}\left(B_{1}^{\prime}\right)_{-\beta}(K) X^{K} \sum_{d \leq K}\left(A_{1}^{\prime}\right)_{\beta}(d+M) \\
& =X^{-M \beta} \sum_{K}\left(B_{1}^{\prime}\right)_{-\beta}(K) X^{K}\left(\left(\left(A_{1}^{\prime}\right)_{\beta}\right)^{+}(K+M)-\left(\left(A_{1}^{\prime}\right)_{\beta}\right)^{+}(M-1)\right)
\end{aligned}
$$

This may be rewritten as

$$
\begin{aligned}
X^{-M \beta} & \sum_{K}\left(B_{1}^{\prime}\right)_{-\beta}(K) X^{K}\left(\left(A_{1}^{\prime}\right)_{\beta}\right)^{+}(K+M) \\
& -X^{-M \beta}\left(\left(A_{1}^{\prime}\right)_{\beta}\right)^{+}(M) Z\left(\left(B_{1}^{\prime}\right)_{-\beta}\right)+X^{-M \beta}\left(A_{1}^{\prime}\right)_{\beta}(M) Z\left(\left(B_{1}^{\prime}\right)_{-\beta}\right) .
\end{aligned}
$$

Thus, altogether we have

$$
\begin{aligned}
S_{L}^{+}= & \sum_{M, \ell, r} A_{2}(\ell) B_{2}(M+\ell)\left(A_{1}^{\prime}\right)_{-\alpha}(r+M)\left(\left(B_{1}^{\prime}\right)_{\alpha}\right)^{+}(r) X^{\ell+r+M+M \alpha} \\
& +\sum_{M, \ell} A_{2}(\ell) B_{2}(M+\ell)\left(X^{-M \beta} \sum_{K}\left(B_{1}^{\prime}\right)_{-\beta}(K) X^{K}\left(\left(A_{1}^{\prime}\right)_{\beta}\right)^{+}(K+M)\right. \\
& \left.-X^{-M \beta}\left(\left(A_{1}^{\prime}\right)_{\beta}\right)^{+}(M) Z\left(\left(B_{1}^{\prime}\right)_{-\beta}\right)+X^{-M \beta}\left(A_{1}^{\prime}\right)_{\beta}(M) Z\left(\left(B_{1}^{\prime}\right)_{-\beta}\right)\right) X^{\ell+M} \\
& -\sum_{M, \ell, d} A_{2}(\ell) B_{2}(M+\ell) A_{1}^{\prime}(d+M) B_{1}^{\prime}(d) X^{\ell+d+M} .
\end{aligned}
$$

In the first line notice that $\left(\left(\left(B_{1}^{\prime}\right)_{\alpha}\right)^{+}\right)_{-\alpha}=B_{1}^{\prime} \cup\{-\alpha\}$. Also, recall our notation:

$$
\mathcal{F}(A, B ; C, D)=\sum_{K, L, M} A(K) B(K+M) C(L) D(L+M) X^{K+L+M} .
$$

Using this notation we have that

$$
\begin{aligned}
S_{L}^{+}= & \mathcal{F}\left(B_{1}^{\prime} \cup\{-\alpha\}, A_{1}^{\prime} ; A_{2}, B_{2}\right)+\mathcal{F}\left(B_{1}^{\prime}, A_{1}^{\prime} \cup\{-\beta\} ; A_{2}, B_{2}\right)-\mathcal{F}\left(B_{1}^{\prime}, A_{1}^{\prime} ; A_{2}, B_{2}\right) \\
& -Z\left(\left(B_{1}^{\prime}\right)_{-\beta}\right) \mathcal{C}\left(A_{1}^{\prime} \cup A_{2} \cup\{-\beta\}, B_{2}\right)+Z\left(\left(B_{1}^{\prime}\right)_{-\beta}\right) \mathcal{C}\left(A_{1}^{\prime} \cup A_{2}, B_{2}\right) .
\end{aligned}
$$

We add this with our expression for $S_{L}^{-}$and have

$$
S_{L}=\mathcal{F}\left(B_{1}^{\prime} \cup\{-\alpha\}, A_{1}^{\prime} ; A_{2}, B_{2}\right)+\mathcal{F}\left(B_{1}^{\prime}, A_{1}^{\prime} \cup\{-\beta\} ; A_{2}, B_{2}\right)-\mathcal{F}\left(B_{1}^{\prime}, A_{1}^{\prime} ; A_{2}, B_{2}\right) .
$$

The expression for $S_{R}$ is obtained by the symmetry $\alpha \leftrightarrow \beta ; A_{1} \leftrightarrow B_{1}$; and $A_{2} \leftrightarrow B_{2}$. Thus,

$$
S_{R}=\mathcal{F}\left(A_{1}^{\prime} \cup\{-\beta\}, B_{1}^{\prime} ; B_{2}, A_{2}\right)+\mathcal{F}\left(A_{1}^{\prime}, B_{1}^{\prime} \cup\{-\alpha\} ; B_{2}, A_{2}\right)-\mathcal{F}\left(A_{1}^{\prime}, B_{1}^{\prime} ; B_{2}, A_{2}\right) .
$$

Recall that

$$
\mathcal{F}(A, B ; C, D)+\mathcal{F}(B, A ; D, C)=\mathcal{C}(A \cup D, B \cup C)+\mathcal{C}(A, B) C(C, D) .
$$

Thus,

$$
\begin{aligned}
S_{L}+S_{R}= & \mathcal{C}\left(A_{1}^{\prime} \cup A_{2} \cup\{-\beta\}, B_{1}^{\prime} \cup B_{2}\right)+C\left(A_{1}^{\prime} \cup A_{2}, B_{1}^{\prime} \cup B_{2} \cup\{-\alpha\}\right) \\
& -C\left(A_{1}^{\prime} \cup A_{2}, B_{1}^{\prime} \cup B_{2}\right)+\mathcal{C}\left(A_{1}^{\prime} \cup\{-\beta\}, B_{1}^{\prime}\right) \mathcal{C}\left(B_{2}, A_{2}\right) \\
& +\mathcal{C}\left(A_{1}^{\prime}, B_{1}^{\prime} \cup\{-\alpha\}\right) \mathcal{C}\left(B_{2}, A_{2}\right)-\mathcal{C}\left(A_{1}^{\prime}, B_{1}^{\prime}\right) \mathcal{C}\left(B_{2}, A_{2}\right) .
\end{aligned}
$$


Adding this to $-S_{0}$ we have

$$
\begin{aligned}
S_{L}+S_{R}-S_{0}= & \mathcal{C}\left(A_{1}^{\prime} \cup A_{2} \cup\{-\beta\}, B_{1}^{\prime} \cup B_{2}\right)+C\left(A_{1}^{\prime} \cup A_{2}, B_{1}^{\prime} \cup B_{2} \cup\{-\alpha\}\right) \\
& -C\left(A_{1}^{\prime} \cup A_{2}, B_{1}^{\prime} \cup B_{2}\right) .
\end{aligned}
$$

This is equal to

$$
\left(1-X^{1-\alpha-\beta}\right) \mathcal{C}\left(A_{1}^{\prime} \cup A_{2} \cup\{-\beta\}, B_{1}^{\prime} \cup B_{2} \cup\{-\alpha\}\right)
$$

as desired.

\section{Proof of Theorem 2}

We shall it convenient to recast the identity of Theorem 2 using a set-theoretic language.

\subsection{A reformulation of the identity}

We begin with 4 sets $A, B, C$ and $D$ and 4 numbers $\alpha, \beta, \gamma$ and $\delta$. We consider

$$
\sum_{\min (M, N)=0} X^{-M(\gamma+\beta)-N(\alpha+\delta)} \Sigma_{1}(M, N) \Sigma_{2}(M, N) X^{M+N}
$$

where

$$
\begin{aligned}
\Sigma_{1}(M, N)= & \sum_{\substack{d, j, k \\
q \leq 1}}(-1)^{q} X^{d(\alpha+\beta)} A_{-\alpha}(j+q+d-\min (q+d, N)) \\
& \times B_{-\beta}(k+q+d-\min (q+d, M)) X^{2 q+d+j+k-\min (q+d, M)-\min (q+d, N)}
\end{aligned}
$$

and

$$
\begin{aligned}
\Sigma_{2}(M, N)= & \sum_{\substack{d, j, k \\
q \leq 1}}(-1)^{q} X^{d(\gamma+\delta)} C_{-\gamma}(j+q+d-\min (q+d, M)) \\
& \times D_{-\delta}(k+q+d-\min (q+d, N)) X^{2 q+d+j+k-\min (q+d, M)-\min (q+d, N)} .
\end{aligned}
$$

The problem is to express this quantity in terms of the $\mathcal{C}$ function, namely we want to prove that the above is

$$
=\left(1-X^{1-\alpha-\beta}\right)\left(1-X^{1-\gamma-\delta}\right) \mathcal{C}(A \cup C \cup\{-\beta,-\delta\}, B \cup D \cup\{-\alpha,-\gamma\}) .
$$

\subsection{Initial reductions}

We can decompose the sum over $M$ and $N$ via

$$
\sum_{\min (M, N)=0} f(M, N)=\sum_{M=0}^{\infty} f(M, 0)+\sum_{N=0}^{\infty} f(0, N)-f(0,0) .
$$

Thus, the sum above is $S_{L}+S_{R}-S_{0}$ where

$$
\begin{gathered}
S_{L}=\sum_{M=0}^{\infty} X^{-M(\gamma+\beta)} \Sigma_{1}(M, 0) \Sigma_{2}(M, 0) X^{M} \\
S_{R}=\sum_{N=0}^{\infty} X^{-N(\alpha+\delta)} \Sigma_{1}(0, N) \Sigma_{2}(0, N) X^{N}
\end{gathered}
$$

and

$$
S_{0}=\Sigma_{1}(0,0) \Sigma_{2}(0,0)
$$


We have

$$
\begin{aligned}
S_{0}= & \left(\sum_{\substack{d, j, k \\
q \leq 1}}(-1)^{q} X^{d(\alpha+\beta)} A_{-\alpha}(j+q+d) B_{-\beta}(k+q+d) X^{2 q+d+j+k}\right) \\
& \times\left(\sum_{\substack{d, j, k \\
q \leq 1}}(-1)^{q} X^{d(\gamma+\delta)} C_{-\gamma}(j+q+d) D_{-\delta}(k+q+d) X^{2 q+d+j+k}\right) .
\end{aligned}
$$

The first factor here is

$$
\begin{aligned}
& \sum_{d, j, k} X^{d(\alpha+\beta)} A_{-\alpha}(j+d) B_{-\beta}(k+d) X^{d+j+k} \\
& \quad-\sum_{d, j, k} X^{d(\alpha+\beta)} A_{-\alpha}(j+1+d) B_{-\beta}(k+1+d) X^{2+d+j+k}
\end{aligned}
$$

which telescopes in $j$ and $k$. Thus, it is

$$
\begin{aligned}
& \sum_{d, j} X^{d(\alpha+\beta)} A_{-\alpha}(j+d) B_{-\beta}(d) X^{d+j}+\sum_{d, k} X^{d(\alpha+\beta)} A_{-\alpha}(d) B_{-\beta}(k+d) X^{d+k} \\
& -\sum_{d} X^{d(\alpha+\beta)} A_{-\alpha}(d) B_{-\beta}(d) X^{d} .
\end{aligned}
$$

The first term here is

$$
\sum_{d, j} X^{d(\alpha+\beta)} A_{-\alpha}(j+d) B_{-\beta}(d) X^{d+j}=\sum_{J=0}^{\infty} A_{-\alpha}(J) X^{J} \sum_{d \leq J} B_{-\beta}(d) X^{d(\alpha+\beta)} .
$$

Now

$$
B_{-\beta}(d) X^{d(\alpha+\beta)}=B_{\alpha}(d)
$$

and

$$
\sum_{d \leq J} B_{\alpha}(d)=\left(B_{\alpha}\right)^{+}(J)
$$

Thus, the above is

$$
\sum_{J=0}^{\infty} A_{-\alpha}(J)\left(B_{\alpha}\right)^{+}(J) X^{J}=\mathcal{C}\left(A_{-\alpha},\left(B_{\alpha}\right)^{+}\right)=\mathcal{C}(A, B \cup\{-\alpha\}) .
$$

The second term is

$$
\mathcal{C}\left(\left(A_{\beta}\right)^{+}, B_{-\beta}\right)=\mathcal{C}(A \cup\{-\beta\}, B)
$$

and the third term is

$$
\mathcal{C}(A, B) \text {. }
$$

We can do the same with the second factor. The net result is that

$$
\begin{aligned}
S_{0}= & (\mathcal{C}(A, B \cup\{-\alpha\})+\mathcal{C}(A \cup\{-\beta\}, B)-\mathcal{C}(A, B)) \\
& \times(\mathcal{C}(C, D \cup\{-\gamma\})+\mathcal{C}(C \cup\{-\delta\}, D)-\mathcal{C}(C, D)) .
\end{aligned}
$$


Next we analyze $S_{L}$. First consider $\Sigma_{1}(M, 0)$ :

$$
\begin{aligned}
\Sigma_{1}(M, 0)= & \sum_{d, j, k} X^{d(\alpha+\beta)} A_{-\alpha}(j+d) B_{-\beta}(k+d-\min (d, M)) X^{d+j+k-\min (d, M)} \\
& -\sum_{d, j, k} X^{d(\alpha+\beta)} A_{-\alpha}(j+1+d) B_{-\beta}(k+1+d-\min (1+d, M)) \\
& \times X^{2+d+j+k-\min (1+d, M)} .
\end{aligned}
$$

We split this into the terms with $d<M$ and those with $d \geq M$. We have

$$
\begin{aligned}
\Sigma_{1}^{-}(M, 0)= & \sum_{\substack{j, k \\
d<M}} X^{d(\alpha+\beta)} A_{-\alpha}(j+d) B_{-\beta}(k) X^{j+k} \\
& -\sum_{\substack{j, k \\
d<M}} X^{d(\alpha+\beta)} A_{-\alpha}(j+1+d) B_{-\beta}(k) X^{1+j+k} \\
= & Z\left(B_{-\beta}\right)\left(\sum_{\substack{j \\
d<M}} X^{d(\alpha+\beta)} A_{-\alpha}(j+d) X^{j}-\sum_{j<M} X^{d(\alpha+\beta)} A_{-\alpha}(j+1+d) X^{1+j}\right) .
\end{aligned}
$$

The sum over $j$ telescopes so that this is

$$
\begin{aligned}
\Sigma_{1}^{-}(M, 0) & =Z\left(B_{-\beta}\right) \sum_{d<M} X^{d(\alpha+\beta)} A_{-\alpha}(d) \\
& =Z\left(B_{-\beta}\right) \sum_{d<M} A_{\beta}(d)=Z\left(B_{-\beta}\right)\left(A_{\beta}\right)^{+}(M-1)
\end{aligned}
$$

Next we consider

$$
\begin{aligned}
\Sigma_{1}^{+}(M)= & \sum_{\substack{j, k \\
d \geq M}} X^{d(\alpha+\beta)} A_{-\alpha}(j+d) B_{-\beta}(k+d-M) X^{d+j+k-M} \\
& -\sum_{\substack{j, k \\
d \geq M}} X^{d(\alpha+\beta)} A_{-\alpha}(j+1+d) B_{-\beta}(k+1+d-M) X^{2+d+j+k-M} .
\end{aligned}
$$

We replace $d$ by $d+M$ and have

$$
\begin{aligned}
\Sigma_{1}^{+}(M)= & \sum_{j, k, d} X^{(d+M)(\alpha+\beta)} A_{-\alpha}(j+d+M) B_{-\beta}(k+d) X^{d+j+k} \\
& -\sum_{j, k, d} X^{(d+M)(\alpha+\beta)} A_{-\alpha}(j+1+d+M) B_{-\beta}(k+1+d) X^{2+d+j+k} .
\end{aligned}
$$

Now the sum over $j$ and $k$ telescopes and we have

$$
\begin{aligned}
\Sigma_{1}^{+}(M)= & \sum_{j, d} X^{(d+M)(\alpha+\beta)} A_{-\alpha}(j+d+M) B_{-\beta}(d) X^{d+j} \\
& +\sum_{k, d} X^{(d+M)(\alpha+\beta)} A_{-\alpha}(d+M) B_{-\beta}(k+d) X^{d+k} \\
& -\sum_{d} X^{(d+M)(\alpha+\beta)} A_{-\alpha}(d+M) B_{-\beta}(d) X^{d}
\end{aligned}
$$


We recognize a convolution in the first term and rewrite this as

$$
\begin{aligned}
\Sigma_{1}^{+}(M)= & \sum_{r} X^{M(\alpha+\beta)} A_{-\alpha}(r+M)\left(B_{\alpha}\right)^{+}(r) X^{r} \\
& +\sum_{k, d} X^{(d+M)(\alpha+\beta)} A_{-\alpha}(d+M) B_{-\beta}(k+d) X^{d+k} \\
& -\sum_{d} X^{(d+M)(\alpha+\beta)} A_{-\alpha}(d+M) B_{-\beta}(d) X^{d}
\end{aligned}
$$

The middle term here may be written as

$$
\begin{aligned}
& \sum_{K} B_{-\beta}(K) X^{K} \sum_{d \leq K} A_{\beta}(d+M) \\
& \quad=\sum_{K} B_{-\beta}(K) X^{K}\left(\left(A_{\beta}\right)^{+}(K+M)-\left(A_{\beta}\right)^{+}(M-1)\right) \\
& \quad=\sum_{K} B_{-\beta}(K)\left(A_{\beta}\right)^{+}(K+M) X^{K}-Z\left(B_{-\beta}\right)\left(A_{\beta}\right)^{+}(M-1) .
\end{aligned}
$$

The second term of this cancels with $\Sigma_{1}^{-}(M, 0)$ and so we have

$$
\begin{aligned}
\Sigma_{1}(M, 0)= & X^{M(\alpha+\beta)} \sum_{K}\left(B_{\alpha}\right)^{+}(K) A_{-\alpha}(K+M) X^{K} \\
& -X^{M(\alpha+\beta)} \sum_{K} B_{\alpha}(K) A_{-\alpha}(K+M) X^{K} \\
& +\sum_{K} B_{-\beta}(K)\left(A_{\beta}\right)^{+}(K+M) X^{K}
\end{aligned}
$$

This may be rewritten as

$$
\begin{aligned}
\Sigma_{1}(M, 0)= & X^{M \beta}\left(\sum_{K}(B \cup\{-\alpha\})(K) A(K+M) X^{K}-\sum_{K} B(K) A(K+M) X^{K}\right. \\
& \left.+\sum_{K} B(K)(A \cup\{-\beta\})(K+M) X^{K}\right)
\end{aligned}
$$

By symmetry

$$
\begin{aligned}
\Sigma_{2}(M, 0)= & X^{M \gamma}\left(\sum_{L}(C \cup\{-\delta\})(L) D(L+M) X^{L}-\sum_{L} C(L) D(L+M) X^{L}\right. \\
& \left.+\sum_{L} C(L)(D \cup\{-\gamma\})(L+M) X^{L}\right) .
\end{aligned}
$$

Recall that we are trying to evaluate

$$
S_{L}=\sum_{M} X^{M(1-\gamma-\beta)} \Sigma_{1}(M, 0) \times \Sigma_{2}(M, 0) .
$$

If we multiply out the three terms of $\Sigma_{1}$ by the three terms of $\Sigma_{2}$ and then sum over $M$ we get a total of nine expressions the first of which is

$$
\begin{aligned}
& \sum_{K, L, M}(B \cup\{-\alpha\})(K) A(K+M)(C \cup\{-\delta\})(L) D(L+M) X^{K+L+M} \\
& \quad=\mathcal{F}(B \cup\{-\alpha\}, A ; C \cup\{-\delta\}, D) .
\end{aligned}
$$


Thus we now see $S_{L}$ as a sum of nine terms of $\mathcal{F}$ at different arguments which we encapsulate in the following table for $S_{L}$ :

\begin{tabular}{ccccc} 
\# sign & $K$ & $K+M$ & $L$ & $L+M$ \\
\hline $1+B \cup\{-\alpha\}$ & $A$ & $C \cup\{-\delta\}$ & $D$ \\
$2-B \cup\{-\alpha\}$ & $A$ & $C$ & $D$ \\
$3+B \cup\{-\alpha\}$ & $A$ & $C$ & $D \cup\{-\gamma\}$ \\
$4-$ & $B$ & $A$ & $C \cup\{-\delta\}$ & $D$ \\
$5+$ & $B$ & $A$ & $C$ & $D$ \\
$6-$ & $B$ & $A$ & $C$ & $D \cup\{-\gamma\}$ \\
$7+$ & $B$ & $A \cup\{-\beta\}$ & $C \cup\{-\delta\}$ & $D$ \\
$8-$ & $B$ & $A \cup\{-\beta\}$ & $C$ & $D$ \\
$9+$ & $B$ & $A \cup\{-\beta\}$ & $C$ & $D \cup\{-\gamma\}$
\end{tabular}

Note that $S_{R}$ is just the same as $S_{L}$ but with $\alpha \leftrightarrow \beta ; \gamma \leftrightarrow \delta ; A \leftrightarrow B$; and $C \leftrightarrow D$. Thus, we have the table for $S_{R}$ :

\begin{tabular}{ccccc} 
\# sign & $K$ & $K+M$ & $L$ & $L+M$ \\
\hline $1+A \cup\{-\beta\}$ & $B$ & $D \cup\{-\gamma\}$ & $C$ \\
$2-A \cup\{-\beta\}$ & $B$ & $D$ & $C$ \\
$3+A \cup\{-\beta\}$ & $B$ & $D$ & $C \cup\{-\delta\}$ \\
$4-$ & $A$ & $B$ & $D \cup\{-\gamma\}$ & $C$ \\
$5+$ & $A$ & $B$ & $D$ & $C$ \\
$6-$ & $A$ & $B$ & $D$ & $C \cup\{-\delta\}$ \\
$7+$ & $A$ & $B \cup\{-\alpha\}$ & $D \cup\{-\gamma\}$ & $C$ \\
$8-$ & $A$ & $B \cup\{-\alpha\}$ & $D$ & $C$ \\
$9+$ & $A$ & $B \cup\{-\alpha\}$ & $D$ & $C \cup\{-\delta\}$
\end{tabular}

Now we pair up line $x$ from $S_{L}$ with line $10-x$ from $S_{R}$ and we use the lemma to express the sum of the $\mathcal{F}$-terms as $\mathcal{C}$ 's. We have

$$
\begin{aligned}
S_{L}+S_{R}= & \mathcal{C}(A \cup C \cup\{-\delta\}, B \cup D \cup\{-\alpha\})+\mathcal{C}(A \cup\{-\beta\}, B) \mathcal{C}(D \cup\{-\gamma\}, C) \\
& -\mathcal{C}(A \cup C, B \cup D \cup\{-\alpha\})-\mathcal{C}(A \cup\{-\beta\}, B) \mathcal{C}(D, C) \\
& +\mathcal{C}(A \cup C, B \cup D \cup\{-\alpha,-\gamma\})+\mathcal{C}(A \cup\{-\beta\}, B) \mathcal{C}(D, C \cup\{-\delta\}) \\
& -\mathcal{C}(A \cup C \cup\{-\delta\}, B \cup D)-\mathcal{C}(A, B) \mathcal{C}(D \cup\{-\gamma\}, C) \\
& +\mathcal{C}(A \cup C, B \cup D)+\mathcal{C}(A, B) \mathcal{C}(D, C) \\
& -\mathcal{C}(A \cup C, B \cup D \cup\{-\gamma\})-\mathcal{C}(A, B) \mathcal{C}(D, C \cup\{-\delta\}) \\
& +\mathcal{C}(A \cup C \cup\{-\beta,-\delta\}, B \cup D)+\mathcal{C}(A, B \cup\{-\alpha\}) \mathcal{C}(D \cup\{-\gamma\}, C) \\
& -\mathcal{C}(A \cup C \cup\{-\beta\}, B \cup D)-\mathcal{C}(A, B \cup\{-\alpha\}) \mathcal{C}(D, C) \\
& +\mathcal{C}(A \cup C \cup\{-\beta\}, B \cup D \cup\{-\gamma\})+\mathcal{C}(A, B \cup\{-\alpha\}) \mathcal{C}(D, C \cup\{-\delta\}) .
\end{aligned}
$$

When we subtract $S_{0}$ all of the terms that are products of two $\mathcal{C}_{\mathrm{s}}$ cancel:

$$
\begin{aligned}
S_{L}+S_{R}-S_{0}= & \mathcal{C}(A \cup C \cup\{-\delta\}, B \cup D \cup\{-\alpha\})-\mathcal{C}(A \cup C, B \cup D \cup\{-\alpha\}) \\
& +\mathcal{C}(A \cup C, B \cup D \cup\{-\alpha,-\gamma\})-\mathcal{C}(A \cup C \cup\{-\delta\}, B \cup D) \\
& +\mathcal{C}(A \cup C, B \cup D)-\mathcal{C}(A \cup C, B \cup D \cup\{-\gamma\}) \\
& +\mathcal{C}(A \cup C \cup\{-\beta,-\delta\}, B \cup D)-\mathcal{C}(A \cup C \cup\{-\beta\}, B \cup D) \\
& +\mathcal{C}(A \cup C \cup\{-\beta\}, B \cup D \cup\{-\gamma\}) .
\end{aligned}
$$




\subsection{The final reckoning}

\section{A generalization of}

$$
A^{+}(d)=A(d)+A^{+}(d-1)
$$

is

$$
(A \cup\{-\alpha\})(d-1)=X^{\alpha}((A \cup\{-\alpha\})(d)-A(d)) .
$$

We apply this to the expression

$$
\left(1-X^{1-\alpha-\beta}\right)\left(1-X^{1-\gamma-\delta}\right) \sum_{r=0}^{\infty}(A \cup C \cup\{-\beta,-\delta\})(r)(B \cup D \cup\{-\alpha,-\gamma\})(r) X^{r}
$$

and after some work find that it is equal to the expression above for $S_{L}+S_{R}-S_{0}$.

\section{Author details}

${ }^{1}$ American Institute of Mathematics, 600 E. Brokaw Rd., San Jose, CA 95112, USA, ${ }^{2}$ School of Mathematics, University of Bristol, Bristol BS8 1TW, UK.

\section{Acknowledgements}

We gratefully acknowledge support under EPSRC Programme Grant EP/K034383/1 LMF: L-Functions and Modular Forms. Research of the first author was also supported by the American Institute of Mathematics and by a grant from the National Science Foundation. JPK is grateful for the following additional support: a grant from the Leverhulme Trust, a Royal Society Wolfson Research Merit Award, a Royal Society Leverhulme Senior Research Fellowship, and a grant from the Air Force Office of Scientific Research, Air Force Material Command, USAF (number FA8655-10-1-3088). He is also pleased to thank the American Institute of Mathematics for hospitality during a visit where this work started.

Received: 22 March 2016 Accepted: 31 August 2016

Published online: 21 November 2016

\section{References}

1. Bogomolny, E.B., Keating, J.P.: Random matrix theory and the Riemann zeros I: three- and four-point correlations. Nonlinearity 8, 1115-1131 (1995)

2. Bogomolny, E.B., Keating, J.P.: Random matrix theory and the Riemann zeros II: $n$-point correlations. Nonlinearity 9 911-935 (1996)

3. Conrey, J.B., Farmer, D.W., Keating, J.P., Rubinstein, M.O., Snaith, N.C.: Integral moments of L-functions. Proc. Lond. Math. Soc. 91, 33-104 (2005)

4. Conrey, J.B., Gonek, S.M.: High moments of the Riemann zeta-function. Duke Math. J. 107(3), 577-604 (2001)

5. Conrey, J. B., Keating, J. P.: Moments of zeta and correlations of divisor-sums: I. Phil. Trans. R. Soc. A 373, 20140313 (2015). arXiv:1506.06842

6. Conrey, J. B., Keating, J. P.: Moments of zeta and correlations of divisor-sums: II. In: Alaca A., Alaca S., Williams K.S. (eds.) Advances in the Theory of Numbers, Proceedings of the Thirteenth Conference of the Canadian Number Theory Association, Fields Institute Communications. pp. 75-85. Springer (2015). arXiv:1506.06843

7. Conrey, J. B., Keating, J. P.: Moments of zeta and correlations of divisor-sums: III. Indagationes Mathematicae. 26(5):736747 (2015). arXiv:1506.06844

8. Duke, W., Friedlander, J.B., Iwaniec, H.: A quadratic divisor problem. Invent. Math. 115(2), 209-217 (1994)

9. Goldston, D.A., Gonek, S.M.: Mean value theorems for long Dirichlet polynomials and tails of Dirichlet series. Acta Arith. 84(2), 155-192 (1998)

10. Keating, J.P., Snaith, N.C.: Random matrix theory and $\zeta(1 / 2+i t)$. Commun. Math. Phys. 214(1), 57-89 (2000)

\section{Submit your manuscript to a SpringerOpen ${ }^{\circ}$ journal and benefit from:}

- Convenient online submission

- Rigorous peer review

- Immediate publication on acceptance

- Open access: articles freely available online

- High visibility within the field

- Retaining the copyright to your article

Submit your next manuscript at springeropen.com 